\title{
On Baroclinic Instability over Continental Shelves: Testing the Utility of Eady-Type Models
}

\author{
SHIH-NAN CHEN AND CHIOU-JiU CHEN \\ Institute of Oceanography, National Taiwan University, Taipei, Taiwan \\ JAMES A. LERCZAK \\ College of Earth, Ocean, and Atmospheric Sciences, Oregon State University, Corvallis, Oregon
}

(Manuscript received 31 July 2019, in final form 12 October 2019)

\begin{abstract}
This study examines the utility of Eady-type theories as applied to understanding baroclinic instability in coastal flows where depth variations and bottom drag are important. The focus is on the effects of nongeostrophy, boundary dissipation, and bottom slope. The approach compares theoretically derived instability properties against numerical model calculations, for experiments designed to isolate the individual effects and justified to have Eady-like basic states. For the nongeostrophic effect, the theory of Stone (1966) is shown to give reasonable predictions for the most unstable growth rate and wavelength. It is also shown that the growing instability in a fully nonlinear model can be interpreted as boundary-trapped Rossby wave interactions - that is, wave phase locking and westward phase tilt allow waves to be mutually amplified. The analyses demonstrate that both the boundary dissipative and bottom slope effects can be represented by vertical velocities at the lower boundary of the unstable interior, via inducing Ekman pumping and slopeparallel flow, respectively, as proposed by the theories of Williams and Robinson (1974; referred to as the Eady-Ekman problem) and Blumsack and Gierasch (1972). The vertical velocities, characterized by a friction parameter and a slope ratio, modify the bottom wave and thus the scale selection. However, the theories have inherent quantitative limitations. Eady-Ekman neglects boundary layer responses that limit the increase of bottom stress, thereby overestimating the Ekman pumping and growth rate reduction at large drag. Blumsack and Gierasch's (1972) model ignores slope-induced horizontal shear in the mean flow that tilts the eddies to favor converting energy back to the mean, thus having limited utility over steep slopes.
\end{abstract}

\section{Introduction}

There are a variety of baroclinic currents on continental shelves or near shelf edges that can develop baroclinic instability. Examples include shelf break jets (Flagg and Beardsley 1978; Gawarkiewicz 1991; Lozier and Reed 2005; Zhang and Gawarkiewicz 2015), river-fed buoyant coastal currents (Qiu et al. 1988; Weingartner et al. 1999; Hetland 2017), tidal mixing fronts (Badin et al. 2009; Brink 2012, 2013), upwelling fronts (Barth 1989; Durski and Allen 2005; Brink 2016a), and boundary currents in marginal seas (Johannessen et al. 1989; Arnone et al. 1990; Blokhina and Afanasyev 2003). Understanding the characteristics of these baroclinically unstable flows, including their growth rate, spatial scale, and parametric sensitivity, has considerable importance because the

\footnotetext{
Corresponding author: Shih-Nan Chen, schen77@ntu.edu.tw
}

instabilities can be a major source of hydrographic variability (e.g., Fratantoni and Pickart 2003) and their eddy fluxes can drive water column restratification (e.g., Spall and Thomas 2016) and cross-margin material exchanges (see Brink 2016b for a review).

To understand the instability properties, the Eady model and its extensions have served as an important theoretical foundation. Aiming at explaining the growth and spatial scales of atmospheric cyclogenesis, Eady (1949) successfully extracted the essence of baroclinic instability by considering a linear stability problem for a thermal wind-balanced flow with constant stratification, vertical shear, and Coriolis parameter. A detailed description of the Eady model is given in section 2. Briefly, with this simple basic flow and under the quasigeostrophic (QG) approximation, the growth rate and wavelength of the most unstable wave mode were derived analytically. The Eady growth rate is governed by 
a Richardson number that is related to the isopycnal slope and thus the amount of potential energy released via horizontal stirring. The wavelength is around 4 times the deformation radius. These predictions were found generally consistent with atmospheric observations. Of equal significance, the simplifications Eady adopted have made the physical processes more transparent. This has subsequently led to mechanistic interpretation of baroclinic instability as mutual reinforcement of Rossby waves (e.g., Bretherton 1966; Hoskins et al. 1985).

After Eady's seminal work, there have been numerous follow-up studies that incorporated additional processes into the Eady theory. Stone $(1966,1971)$ extended the problem to account for nongeostrophic effects- that is, departures from QG due to weak stratification of the basic flow (i.e., steep isopycnals) such that fluid motion is not constrained to be nearly horizontal (see section 2a). Williams and Robinson (1974) proposed a conceptual model that allows boundary dissipation to modify the interior instability by inducing Ekman pumping (see also in Holopainen 1961; Stipa 2004). The effects of topography were considered by Blumsack and Gierasch (1972, hereafter BG72) and Mechoso (1981) who showed that the boundary slopes can exert stabilizing or destabilizing influences, depending upon their orientation relative to the isopycnals. There are other extensions for specific problems, such as adding air-sea fluxes (Spall 2007) and linear horizontal shear to the basic flow (Barcilon and Blumen 1995). Note that the above references only represent a fraction of theoretical developments for baroclinic instability. Thorough reviews and extensive lists of references can be found in Pedlosky (1979) and Pierrehumbert and Swanson (1995). Further descriptions of the extensions of interest in this study are given in section 2 .

In the context of coastal baroclinic instability, the Eady-type models described above have been invoked to inform interpretation and parameterizations. For slope effects, a number of recent studies on unstable flow over topography have reported evidence of slope stabilization in a manner consistent with BG72 (e.g., Isachsen 2011; Pennel et al. 2012). Guided by BG72, the slope dependence was incorporated into the parameterizations of eddy growth rate and scale for tidal mixing fronts (Brink 2012) and of eddy buoyancy fluxes for boundary currents (Spall 2004). Concerning the stability of buoyant coastal currents, Hetland (2017) simulated a range of idealized coastal currents that have Eady-like basic flows on a slope. He found growing baroclinic instability only in a subset of the experiments, contrary to the expectation from BG72 which predicts a finite growth rate for all cases. This contradiction then led Hetland (2017) to propose width limitation as an additional stabilizing factor. Concerning frictional effects, Brink and Cherian (2013) found bottom drag to reduce the eddy kinetic energy (EKE) in unstable tidal mixing fronts. As in Williams and Robinson (1974), they hypothesized that the eddy field spins down due to Ekman pumping. This led them to develop a parameterization of frictionally damped EKE that depends on a ratio of eddy turnover and spindown time.

The above examples demonstrate that Eady-type theories provide useful guidance for understanding baroclinic instability on continental shelves. However, the limitations of these theories have not been fully examined. Foremost, quantitative evaluations of Eadytype theories are rare, at least for coastal flows. It is therefore hard to determine their limitations and why differences may occur. The theories also made assumptions that may be questionable in coastal settings where depth variations and bottom drag are of leadingorder importance. For example, BG72 considered a mild slope (see section 2a) over which the mean flow is assumed horizontally uniform. It is not clear if depth changes in shallow flows could induce significant horizontal shear (via thermal wind; see section 5) and thus affect the instability. To model the frictional influences, Williams and Robinson (1974) assumed that all dissipative effects in a thin bottom Ekman layer can be represented by Ekman pumping that forces the unstable interior flow (see section 2a). This conceptual model is largely untested. Moreover, in shallow flows, all of the factors described above, including bottom drag, bottom slope, nongeostrophy, and horizontal shear, most likely coexist (e.g., Brink and Cherian 2013). The interplays between these factors make interpreting the responses of baroclinic instability difficult. Hence, there is a need to understand the individual effects before one can appreciate the combined influences.

In this study we examine the utility and limitations of Eady-type theories as applied to baroclinic instability in coastal flows where depth changes and bottom drag are significant. An overall goal is to better understand the individual effects of nongeostrophy, boundary dissipation, and bottom slope. We focus on these three because of their common presence and because their individual effects have been previously incorporated into the Eady model, thereby providing theoretical bases to compare with. Combined effects are left for future studies. Here, the approach is to test the theoretically derived instability properties against calculations from numerical experiments. The experiments are designed to isolate the three effects on baroclinic instability, with a parameter range and basic flows guided by a separate set of idealized simulations of unstable coastal currents. This paper is organized as follows: In section 2, we describe 
(a)

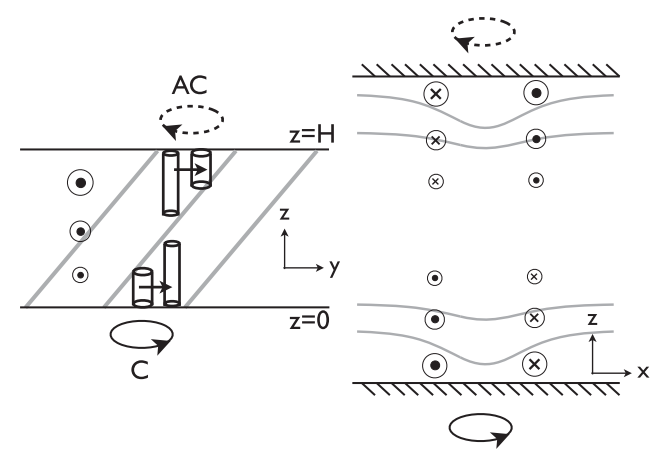

(c) mean flow $U \quad c_{\text {top }}=-U / 2$

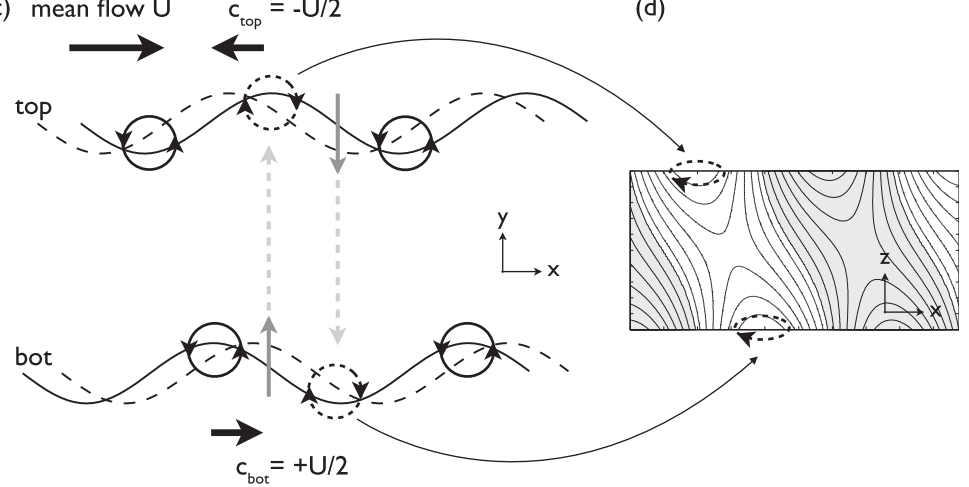

FIG. 1. Schematics of Rossby wave interactions in the Eady model. (a),(b) The generation of boundary-trapped Rossby waves is illustrated. (c),(d) The wave phase locking and phase relation are shown. Shown in (a) is the cross-shore structure of an Eady basic flow that has uniformly spaced and sloped density surfaces (gray line) and a constant vertical shear. Because we examine the Eady-type theories with applications to coastal current instability in mind, throughout this study we refer to the direction of the basic flow as the alongshore $(+x)$ direction and the directions perpendicular to the mean flow as the offshore $(+y)$ and onshore $(-y)$ directions (see Fig. 4$)$. With the basic flow in (a), an offshore displacement of a fluid element along the upper (lower) boundary would induce anticyclonic (AC) [cyclonic (C)] circulation. The illustration in (b) shows that a positive buoyancy anomaly due to offshore advection, combined with thermal wind, drives the same sense of circulation as in (a). In (c), for the top wave, the buoyancy advection by the induced circulation leads to a shift of the buoyancy anomalies, manifested as a wave propagating toward the $-x$ direction. An opposite pattern occurs for the bottom wave. The counterpropagating waves (thick black arrows), advected by a vertically sheared mean flow, can achieve phase locking. Both waves then propagate in the $+x$ direction at the same apparent speed of $U / 2$. When the bottom wave leads the top by $\pi / 2$, the induced onshore velocity (gray solid arrow) of the top wave enhances the bottom wave trough (gray dashed arrow), and vice versa. This phase structure allows the waves to reinforce each other. When viewed in the $x-z$ plane as shown in (d), the structure exhibits a westward phase tilt. See main text for details.

the methods and review the theoretical extensions of the three effects. In addition, designs and justification of the numerical experiments are given. In sections $3-5$, we examine the effects of nongeostrophy, boundary dissipation, and bottom slope, respectively. Section 6 provides a summary and discussions of implications.

\section{Methods}

\section{a. Background of linear stability theories}

\section{1) EADY MODEL AND NONGEOSTROPHIC EFFECTS}

The Eady model of baroclinic instability is a standard material in many geophysical fluid dynamics textbooks. A brief review of the mathematical treatment using conservation of quasigeostrophic potential vorticity (QGPV) is given in appendix A. Here we concentrate on its main results and interpretation via Rossby wave interactions.

Following Hoskins et al. (1985), the mechanism of baroclinic instability in the Eady model can be interpreted as the mutual enhancement of two boundarytrapped Rossby waves. The key ingredients are 1) generation of boundary-trapped Rossby waves and 2) wave phase locking and mutual reinforcement such that disturbances can grow. These processes are schematized in Fig. 1. First, Rossby (vorticity) waves can be generated at two horizontal boundaries by cross-shore disturbances (in the $y$ direction). Because Eady basic flow has constant vertical shear, constant stratification, is horizontally uniform, and is on an $f$ plane, there is no cross-shore gradient of background potential vorticity $\left[\partial \bar{q} / \partial y=-\bar{u}_{y y}-\left(f_{0}^{2} / N^{2}\right) \bar{u}_{z z}=0\right.$; an overbar refers to alongshore mean; see appendix $\mathrm{A}]$, and thus cannot support Rossby wave propagation in the interior. However, the density surfaces intersecting top and bottom boundaries provide potential vorticity (PV) gradients necessary for Rossby waves (Bretherton 1966). The wave generation and propagation may be understood via vortex stretching or buoyancy perturbations at the boundaries. For example, in Fig. 1a, an offshore displacement (toward $+y$ ) of a fluid element along the top boundary tends to induce vortex squashing (since vertical velocity $w=0$ at $z=H$ ) and anticyclonic circulation. Alternatively, buoyancy advection associated with an offshore flow gives a local isopycnal depression and thus a positive buoyancy anomaly at the top (Fig. 1b). By thermal wind balance and by requiring the induced velocity to decay toward the interior, the depression again induces an anticyclone trapped at the top boundary. The anticyclone advects the cross-shore buoyancy gradient (i.e., dashed circle in Fig. 1c) and shifts the buoyancy anomaly, manifested as a wave propagating toward the $-x$ direction. Along the bottom boundary, an 
opposite pattern occurs. Therefore, when the basic flow is perturbed, there are two boundary-trapped Rossby waves that propagate in the opposite direction: Top wave moves toward the $-x$ direction, relative to the basic flow, while bottom wave moves toward the $+x$ direction (Fig. 1c).

When the two waves are phase locked and are in certain phase relation to allow for mutual enhancement, the wave amplitude can grow, manifested as the development of baroclinic instability. Phase locking simply means that the two waves move at the same apparent speed to sustain the mutual enhancement. With parallel horizontal boundaries and with a constant isopycnal slope, one expects the waves to have identical intrinsic phase speeds. It can be shown that, if considered separately, each boundary supports a wave whose intrinsic phase speed is $U / \mu$ [where $\mu$ is a dimensionless wavenumber; see appendix A and Vallis (2017, chapter 9.7.2)]. Because the top wave is advected by the mean flow $U$ and the two waves have identical intrinsic phase speed $U / \mu$, the condition of phase locking is $\mu=2$. That is, the top wave translates at phase speed of $-U / 2$ (i.e., toward $-x$ ) but is advected by mean flow $U$. The apparent phase speed is then $U / 2$, equal to that of the bottom wave.

In addition to having an apparent speed of $U / 2$ toward $+x$ direction, the two waves must retain a certain phase relationship, referred to as westward phase tilt (Fig. 1d; see, e.g., Vallis 2017), so that they can amplify each other. As illustrated in Fig. 1c, when the bottom wave leads the top by a quarter wavelength (i.e., $\pi / 2$ phase difference), the induced onshore velocity of the top wave (denoted by the downward gray arrow) would align with the trough of the bottom wave, enhancing the bottom displacement. Similarly, the induced offshore velocity of the bottom wave (denoted by the upward gray arrow) is at the top wave's crest, again enhancing the surface displacement. Viewed from the $x-z$ plane, the wave structure tilts westward with height (Fig. 1d; leaned toward $-x$ direction). It can also be shown that the westward phase tilt favors downgradient buoyancy transport, thereby allowing release of potential energy to fuel the growth of baroclinic instability (see Pedlosky 1979).

The solution of the Eady model reflects the wave properties described above. Specifically, the unstable waves translate toward the $+x$ direction at the phaselocking speed of $U / 2$, and the most unstable wave mode has a top to bottom phase difference of $\pi / 2$, as expected for the westward phase tilt. These comparisons are described in appendix A.

From the Eady solution, the well-known Eady growth rate and wavelength (i.e., the most unstable mode, with cross-shore wavenumber $l=0$; Fig. A1a) are, respectively,

$$
\sigma_{\text {Eady }} / f_{0}=0.31 \mathrm{Ri}^{-1 / 2}, \quad \lambda_{\text {Eady }} / L_{d}=(2 \pi / 1.61) \approx 3.9 .
$$

Here the grow rate $\sigma_{\text {Eady }}$ and wavelength $\lambda_{\text {Eady }}$ are made dimensionless by the Coriolis parameter $f_{0}$ and the deformation radius $L_{d}\left(=N H / f_{0}\right)$, respectively. $\mathrm{Ri}$ is a Richardson number of the mean flow that governs the Eady model:

$$
\mathrm{Ri}=\frac{N^{2}}{(U / H)^{2}}=\frac{N^{2}}{\left(M^{2} / f_{0}\right)^{2}}=\frac{N^{2} f_{0}^{2}}{M^{4}},
$$

where $M^{2}$ is defined as the negative of the offshore buoyancy gradient $(=-\partial \bar{b} / \partial y=$ constant; where $b$ is buoyancy), $N$ is the buoyancy frequency, and $H$ and $U$ are the height and basic state velocity scales, respectively. From Eq. (1), we see that the growth rate varies inversely with $\mathrm{Ri}$. This can be understood by noting that $\mathrm{Ri}$ is related to the isopycnal slope $S$. For fixed $f_{0}$ and $M^{2}$, a smaller Ri corresponds to a smaller $N^{2}$ and hence a steeper isopycnal slope $\left[S \equiv-(\partial \bar{\rho} / \partial y) /(\partial \bar{\rho} / \partial z)=M^{2} / N^{2}\right]$. As shown by Haine and Marshall (1998), the potential energy released by parcel rearrangement is proportional to $\Delta b \Delta z$. For a fixed horizontal excursion $\Delta y$ and constant $M^{2}$, the buoyancy variation $\Delta b$ is fixed. But, a steeper isopycnal slope (i.e., smaller $\mathrm{Ri}$ ) allows a parcel to rise higher (i.e., larger $\Delta z$ ), thereby releasing more potential energy per unit displacement and thus resulting in a greater growth rate.

However, Eady's growth rate is only formally applicable to a quasigeostrophic (QG) flow. As Ri decreases, the small-Rossby-number assumption in QG would eventually break down, and the Eady theory would need to be modified to account for "nongeostrophic" effects. Here the nongeostrophy is due to weak stratification (i.e., steep isopycnals) such that fluid motion is not constrained to a nearly horizontal plane as in QG. To see this breakdown of QG, we can express the Rossby number as an inverse of $\mathrm{Ri}$, by taking $L_{d}$ as the characteristic spatial scale [as suggested by Eq. (1)]: $\mathrm{Ro}=U /\left(f L_{d}\right)=U /(N H)=\mathrm{Ri}^{-0.5}$. As Ri decreases to $O(1)$, Ro also becomes $O(1)$, and the QG approximation is no longer valid.

Stone (1966) made nongeostrophic corrections to the Eady theory. He found an approximate growth rate and wavelength as

$$
\begin{aligned}
\sigma_{\text {Stone }} / f_{0} & \approx 0.304(1+\mathrm{Ri})^{-1 / 2}, \\
\lambda_{\text {Stone }} / L_{d} & \approx 3.97 \sqrt{(1+\mathrm{Ri}) / \mathrm{Ri}} .
\end{aligned}
$$

One can immediately see the similarities between Eqs. (1) and (3). For Ri $\gg 1$ (i.e., QG limit), $(1+\mathrm{Ri})$ 

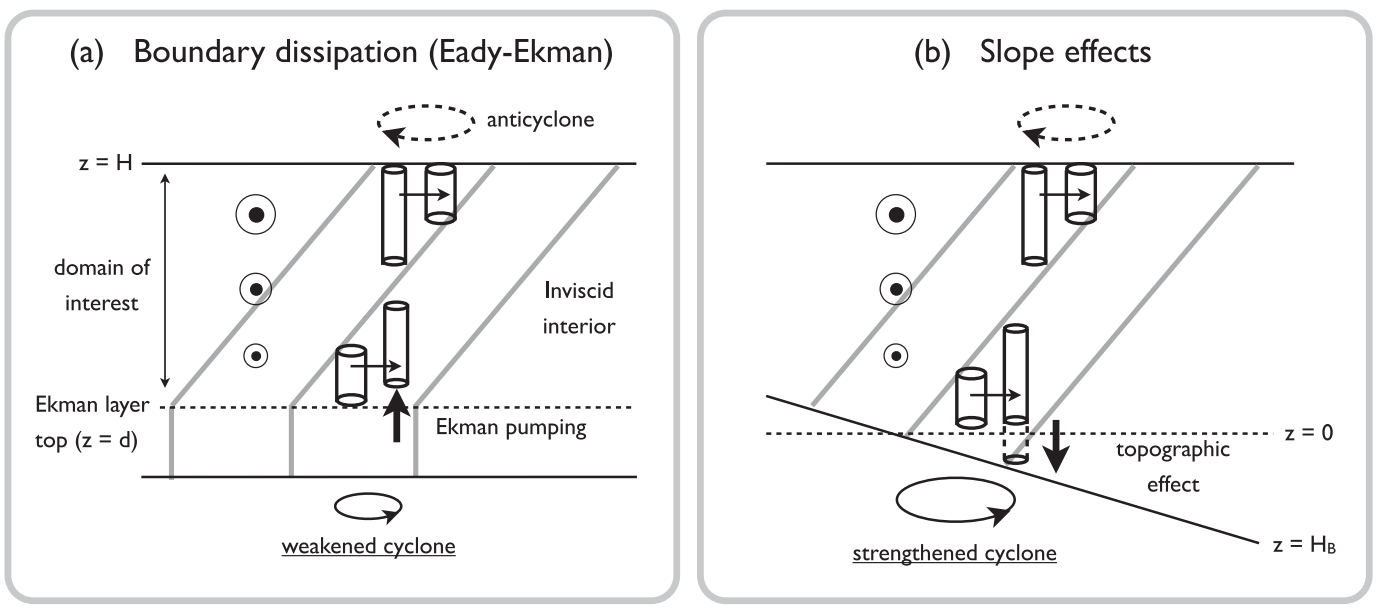

FIG. 2. Schematics demonstrating how (a) boundary dissipation and (b) bottom slope affect the baroclinic instability in the Eady problem. These models are constructed based on the theories of Williams and Robinson (1974; Eady-Ekman) and BG72. In (a), the effect of boundary dissipation is represented by the Ekman pumping (thick black arrow) that serves as a forcing imposed at the lower boundary of the inviscid interior [Eqs. (4) and (5)]. The domain of the linear stability problem is between $z=d$ and $H(d$ is the Ekman layer thickness that is assumed to be thin as compared to the interior). In (b), the presence of a bottom slope modifies the bottom kinematic boundary condition in the Eady problem [Eqs. (8) and (9)]. A negative bottom slope $\left(S_{B}=d H_{B} / d y\right)$ corresponds to water depth increasing offshore. In essence, both effects are represented by vertical velocities at the lower boundary. They then modify the bottom wave and in turn the instability properties.

approaches Ri, and Stone's solution approximates Eady's. When comparing Eqs. (1) and (3), we can see that the nongeostrophic effect tends to reduce the growth rate and selects a longer wavelength. Nakamura (1988) suggested that, as compared to the Eady solution, nongeostrophic effects make the boundary-trapped waves shallower (i.e., decay toward the interior over a shorter vertical scale). The instability therefore tends to select longer waves in order to maintain the vertical coupling. These consequences will be examined in section 3 .

\section{2) EADY-EKMAN PROBLEM: BOUNDARY DISSIPATION EFFECTS}

How would the presence of boundary dissipation modify baroclinc instability? Williams and Robinson (1974) considered such a problem by adding boundary dissipation into the Eady model (termed the EadyEkman problem hereafter). They assume that dissipation due to bottom drag and turbulent stresses is concentrated in a bottom Ekman layer that is thin compared to the interior thickness. One may then represent the dissipative effects via Ekman pumping which modifies the lower boundary condition of the Eady model.

A conceptual interpretation of the Eady-Ekman problem is shown in Fig. 2a. Compared to the inviscid Eady basic flow in Fig. 1a, the uniformly sloped density surfaces in the interior and the horizontal surface boundary remain unchanged, meaning that the governing equations (A1) and (A3a) are intact. There is now an Ekman layer with thickness $d$ at the bottom boundary. We can study the interior flow stability by simply moving the lower boundary to the top of the bottom Ekman layer $(z=d$; dashed line in Fig. 2a) where a new bottom boundary condition is vertical velocity $w$ set by Ekman pumping. If we use a linear friction factor $\gamma$ to relate the bottom stress with the interior geostrophic velocity $\left(\tau_{b x}^{\prime}, \tau_{b y}^{\prime}\right)=\rho_{0} \gamma\left(u_{g}^{\prime}, v_{g}^{\prime}\right)$ (e.g., Brink and Cherian 2013), $w$ can be expressed in terms of interior vorticity as:

$$
w(z=d)=\left(\gamma / f_{0}\right)\left(\partial v^{\prime} / \partial x-\partial u^{\prime} / \partial y\right)=\left(\gamma / f_{0}\right) \nabla^{2} \psi^{\prime},
$$

and buoyancy conservation at the lower boundary $(z=$ d) becomes

$$
\left(\frac{\partial}{\partial t}\right) f_{0} \frac{\partial \psi^{\prime}}{\partial z}-\Lambda f_{0} \frac{\partial \psi^{\prime}}{\partial x}+\frac{N^{2} \gamma}{f_{0}} \nabla^{2} \psi^{\prime}=0
$$

[where $\Lambda\left(=M^{2} / f_{0}\right)$ is the thermal wind shear; see Eq. (A2)]. In Eq. (5) we have assumed that $d$ is sufficiently thin $(d / H \approx 0)$ such that buoyancy advection by the mean flow is negligible [i.e., $U(z=d)=\Lambda d \approx 0$ ]. Equations (A1), (A3a), and (5) form a new equation set that can be solved for unstable wave modes. The solution is given in appendix A, section $\mathrm{b}$. Adding linear Ekman friction introduces a new parameter (see also in Stipa 2004) 


$$
\Delta_{E}=\left(\frac{\gamma}{f_{0}}\right) k / S=(d k) / S,
$$

where $d=\gamma / f_{0}$ may be thought of as a scale for Ekman layer thickness. Below we summarize some of the key results that will be further examined in this study.

Boundary dissipation tends to reduce the instability growth rate and shift the most unstable mode to a longer wavelength. Figure 3 a shows the stability diagram in a parameter space consisting of the dimensionless alongshore wavenumber $\left(\tilde{k}=k L_{d}\right)$ and the friction parameter $\Delta_{E}$. The color contours are the dimensionless growth rate $\left[\sigma /\left(f_{0} \mathrm{Ri}^{-1 / 2}\right)\right]$. Waves that have no cross-shore structure $(l=0)$ are plotted, as they grow faster than those with $l \neq 0$. Note that the friction parameter $\Delta_{E}$ is linearly proportional to the friction factor $\gamma$. As $\Delta_{E}$ increases, the maximal growth rate found following the black curve in Fig. 3a decreases, and the corresponding wavenumber decreases. For example, with $\Delta_{E}=0$, the stability curve reverts to the Eady model, with maximal growth rate $\sigma /\left(f_{0} \mathrm{Ri}^{-1 / 2}\right)$ equal to 0.31 and $\tilde{k}$ of 1.61 [Eq. (1)]. When $\Delta_{E}$ increases to 0.5 , the growth rate decreases by more than $50 \%$ to 0.15 , and $\tilde{k}$ decreases to 1.5 , indicating a shift toward a longer wavelength.

The shift toward longer waves may be understood as a response to a decrease in bottom wave phase speed. In Fig. 3b, we plot the solution of apparent wave speed $c$ for the Eady-Ekman problem (see appendix A) against $\Delta_{E}$. Because there is no mean flow at the bottom, $c$ is equal to the intrinsic bottom wave speed. It can be seen that the bottom wave slows as $\Delta_{E}$ increases. Recall that, when the top and bottom waves are considered separately, the top wave propagates at a speed of $U-U / \tilde{k}$ (i.e., intrinsic propagation at $-U / \tilde{k}$ toward $-x$ direction, advected by mean flow $U$; for $l=0, \mu=\tilde{k}$ ). Since the bottom wave is slowed, the top wave $(-U / \tilde{k})$ has to speed up to remain phase locked. The instability therefore tends to select a longer (smaller $\tilde{k}$ ) and faster wave mode when friction is included.

We may interpret the slowdown of the bottom wave from the perspective of buoyancy perturbations. As described in section 2a and in Fig. 1b, buoyancy anomaly at the boundaries induces circulation that leads to wave propagation. At the bottom boundary, the linearized buoyancy conservation [Eq. (5)] can be expressed as

$$
\frac{\partial b^{\prime}}{\partial t}=M^{2} v^{\prime}-N^{2} w=M^{2} v^{\prime}\left[1-\left(w / v^{\prime}\right) / S\right],
$$

where $w$ in this case represents Ekman pumping. We can see that an offshore displacement $\left(v^{\prime}>0\right)$ favors generation of positive buoyancy anomaly $\left(\partial b^{\prime} / \partial t>0\right)$ and
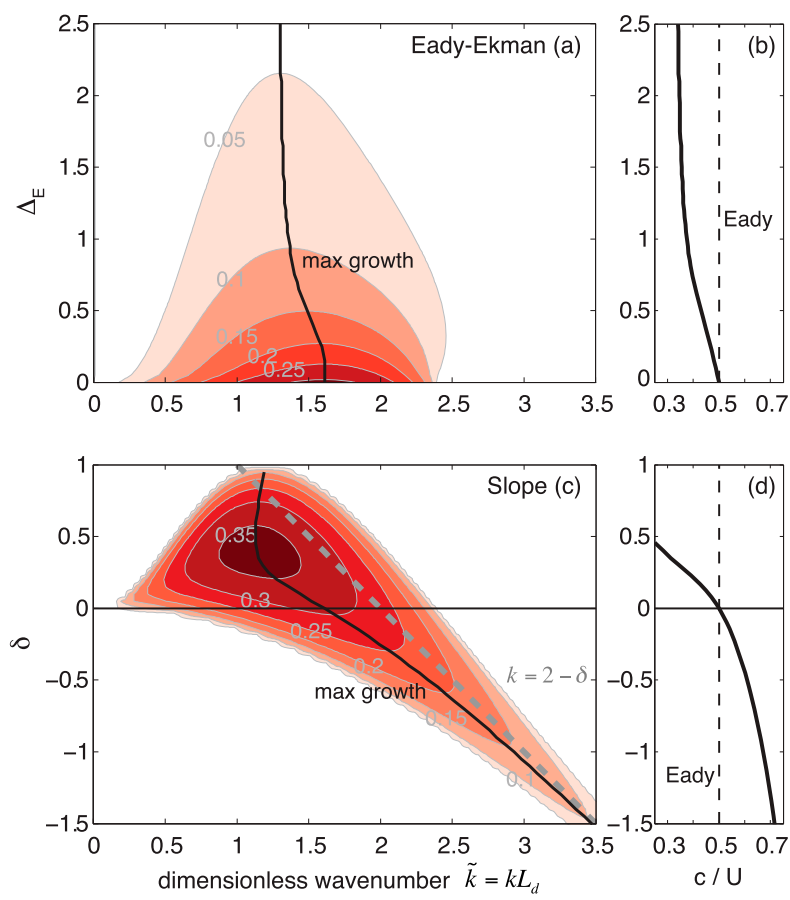

FIG. 3. (a),(c) Stability diagrams for the Eady-Ekman and BG72 theories, respectively, illustrated in Fig. 2 and section 2a. The full solutions are given in the appendix $\mathrm{A}$. The dimensionless growth rate [contoured; $\sigma /\left(f_{0} \mathrm{Ri}^{-0.5}\right)$ ] is shown as a function of dimensionless alongshore wavenumber $\left(\tilde{k}=k L_{d} ; L_{d}\right.$ is the deformation radius) and a friction parameter $\Delta_{E}$ in (a) and a slope ratio $\delta$ in (c) [Eqs. (6) and (10)]. The cross-shore wavenumber $l$ is set to zero. The black curves indicate the most unstable mode for each $\Delta_{E}$ and $\delta$ value. In (c), an approximate marginal stability curve is denoted by the gray dashed line (see section 2a). (b),(d) The apparent wave speed $c$ normalized by the mean flow velocity scale $U$ for the most unstable waves. Because mean flow is zero at the bottom, the apparent speed is equal to the bottom wave speed. The unstable Eady wave speed is $U / 2$.

thus cyclonic circulation along the bottom boundary (Figs. 1a,b). However, in the presence of a bottom Ekman layer, cyclonic circulation drives Ekman pumping $(w>0)$ that favors negative buoyancy anomaly by advecting denser fluid upward. The pumping thus works against horizontal disturbance [i.e., $w / v^{\prime}>0$ in Eq. (7)], acting to reduce the bottom buoyancy perturbations and slow the bottom waves. Note also that, using Eq. (4) and $l=0$, we can express the pumping velocity $w$ as $i d k v^{\prime}$ such that $\left(w / v^{\prime}\right) / S=i(d k / S)=i \Delta_{E}$ (where $i$ is the unit imaginary number). The friction parameter $\Delta_{E}$ thus serves as a measure of pumping strength that affects the bottom parcel trajectory $\left(w / v^{\prime}\right)$ relative to the isopycnal slope $S$ (see section 6a for discussion).

\section{3) EADY PROBLEM ON A SLOPE}

The effects of bottom slope can also be incorporated into the Eady model by modifying the lower boundary 
condition. This problem was treated analytically by BG72 and is schematized in Fig. 2b. In the presence of a bottom slope $S_{B}$ in the cross-shore direction, the kinematic bottom boundary condition is

$$
w(z \approx 0)=S_{B} v^{\prime}=S_{B} \partial \psi^{\prime} / \partial x,
$$

where $S_{B} \equiv d H_{B} / d y$ and $H_{B}(y)$ is the topographic variation with relative to $z=0$. Bottom buoyancy conservation becomes

$$
\left(\frac{\partial}{\partial t}\right) f_{0} \frac{\partial \psi^{\prime}}{\partial z}-\Lambda f_{0} \frac{\partial \psi^{\prime}}{\partial x}+N^{2} S_{B} \frac{\partial \psi^{\prime}}{\partial x}=0 .
$$

Again we find the growth rate of unstable wave modes by solving Eqs. (A1), (A3a), and (9) (see appendix A, section c). Note that $S_{B}$ can be positive or negative. In this study we focus on configurations of $S_{B}<0$ (i.e., depth increasing offshore) and $S=M^{2} / N^{2}>0$ (i.e., isopycnals sloped up offshore) (Fig. 2b), typical for buoyancy-driven coastal flows. Moreover, in BG72, the bottom slope is assumed to be mild in the sense that the depth changes during a parcel displacement are small compared to the mean depth (i.e., QG limit). This requires $\Delta h / H \sim S_{B} L_{d} / H \sim S_{B} N / f_{0} \sim O($ Ro). The mild slope also allows BG72 to neglect horizontal shear in the mean flow [i.e., $U=\Lambda z$ in Eq. (A1)]. This turns out to have nonnegligible influences on the unstable wave properties over a steep slope (see section 5).

The stability diagram indicates that increasing bottom slope (more negative $S_{B}$ ) reduces the growth rate and shifts the instability to a shorter wavelength. In Fig. 3c, the dimensionless growth rate with $l=0$ is plotted [using Eq. (A8)] versus the alongshore wavenumber $\tilde{k}$ and the key dimensionless parameter

$$
\delta=S_{B} /\left(M^{2} / N^{2}\right)=S_{B} / S,
$$

which measures the bottom steepness relative to the isopycnal slope $S$. The curve along $\delta=0$ again corresponds to the Eady model. Here we focus on the lower half of the parameter space $(\delta \leq 0)$ where the bottom and isopycnals are sloped in the opposite direction. It can be seen that, as $|\delta|$ increases, the growth rate of the most unstable mode (denoted by the black curve) decreases, and the wavenumber increases. For example, when compared to the Eady model, at $\delta=-0.5$ the growth rate reduces from 0.31 to 0.215 while the wavenumber increases from 1.61 to 2.33 , indicating a shift to shorter wave modes.

The scale selection may again be understood from a change in bottom wave characteristics. Figure $3 \mathrm{~d}$ shows the response of bottom wave speed to $\delta$ variations for the most unstable mode. As the bottom slope steepens (more negative $\delta$ ), the bottom wave speeds up. To maintain the phase lock, the top wave must then slow down. The instability therefore tends to select a shorter and slower wave mode. To understand the speedup of the bottom waves, we return to the bottom buoyancy conservation:

$$
\begin{aligned}
\frac{\partial b^{\prime}}{\partial t} & =M^{2} v^{\prime}-S_{B} N^{2} v^{\prime}=M^{2} v^{\prime}\left(1-\frac{S_{B}}{M^{2} / N^{2}}\right) \\
& =M^{2} v^{\prime}(1-\delta),
\end{aligned}
$$

where we have used Eq. (8) to express the bottom vertical velocity. We see in Eq. (11) that, for a given crossshore velocity $v^{\prime}$, increasing bottom slope enhances the bottom buoyancy perturbations and hence the wave speed by allowing the slope-parallel flow to cross more isopycnals. It can be shown that, when the boundarytrapped waves are considered separately as before, the bottom wave speed on a slope is $U(1-\delta) / \tilde{k}$. To match the top wave speed of $U-U / \tilde{k}$, the wavenumber needs to increase with the slope as $\tilde{k}=2-\delta$ (e.g., BG72; Pedlosky 2016). This simple relation of matching wave speed is shown as the gray dashed line in Fig. 3c. It indeed approximately captures the tendency of instability shifting toward shorter waves.

\section{b. Numerical model}

We use a primitive equation ocean model Regional Ocean Modeling System (ROMS; Haidvogel et al. 2000; Shchepetkin and McWilliams 2005) to test the utility of the Eady-type theories described above. The parameter ranges are chosen to be suited for buoyant coastal currents (see examples below), but the results are expected to be generic. The approach is to compare the theoretically derived growth rate and wave properties against ROMS calculations. ROMS has been used to study instabilities in a variety of coastal flows (see introduction). Below we use examples of coastal current simulations to help constrain and motivate the problem. We then describe the experiments that isolate the effects of nongeostrophy, boundary dissipation and bottom slope.

Figures $4 a-c$ show the structure of an unstable buoyant coastal current generated by a coastal discharge. The simulation was carried out in a $600 \mathrm{~km}$ (alongshore: $x$ ) $\times$ $210 \mathrm{~km}$ (cross-shore: $y$ ) channel, with a uniform bottom slope of $10^{-3}$, uniform horizontal grid spacing of $520 \mathrm{~m}$, and 30 sigma levels. It was forced only by an inflow (20 km wide centered at $x=0$ ) with a salinity anomaly of 2 psu and freshwater flux of $4000 \mathrm{~m}^{3} \mathrm{~s}^{-1}$. Temperature is set constant in this study. The boundary conditions follow Chen and Chen (2017). The $k-\varepsilon$ turbulence closure was employed (see Warner et al. 2005). Throughout this work, explicit horizontal viscosity and diffusivity are set 

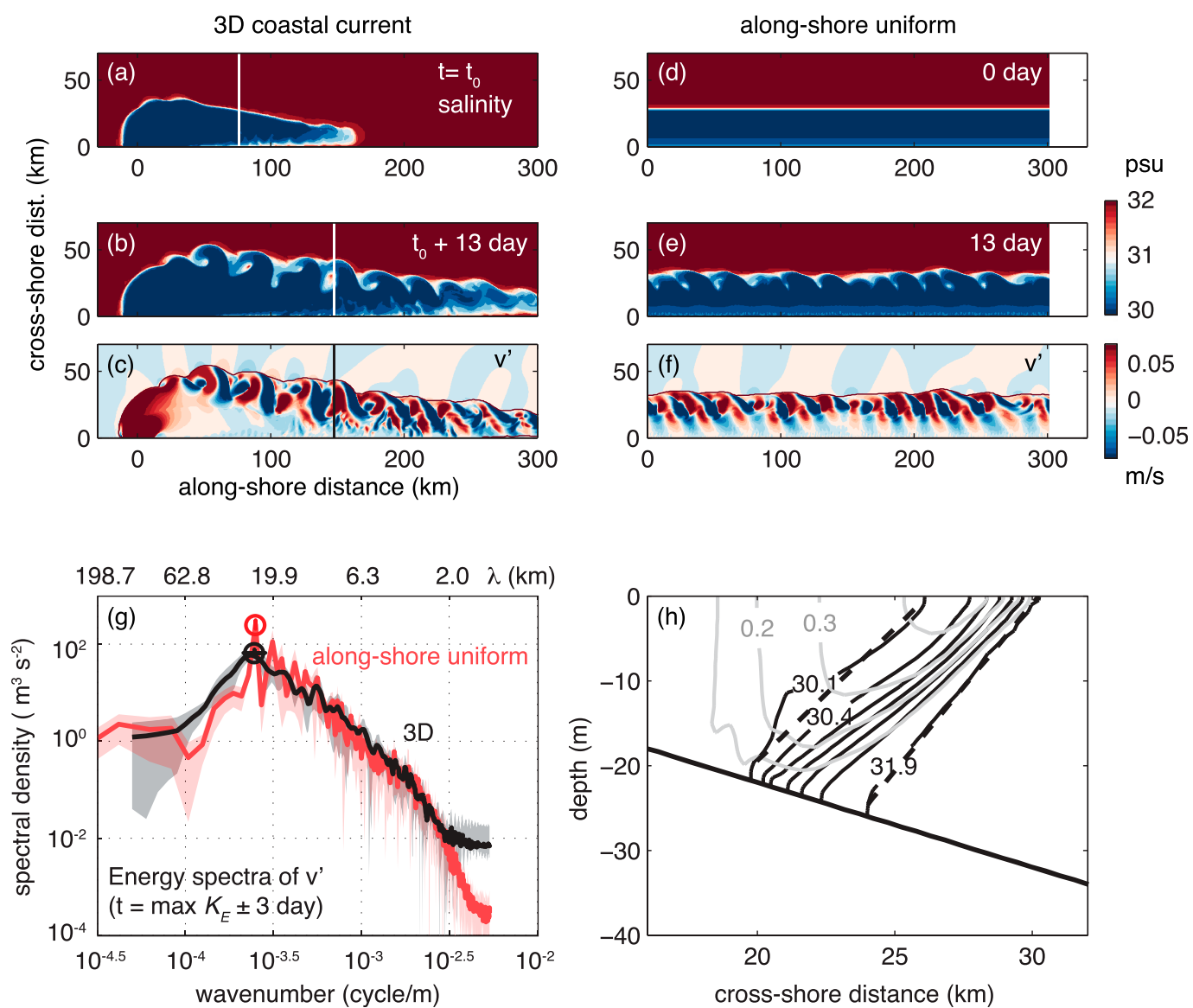

FIG. 4. Snapshots of (a)-(c) three-dimensional (3D) and (d)-(f) alongshore uniform (2D) simulations of buoyant coastal currents. The 2D basic flow in (d) is constructed by averaging the cross-shore salinity profiles around the center of the 3D coastal current in (a) (i.e., $x \sim 80 \mathrm{~km}$; denoted by the vertical white line). In (a), (b), (d), and (e), the color contour indicates surface salinity, while (c) and (f) show the surface cross-shore velocity $v^{\prime}$. The snapshots in (b), (c), (e), (f) are taken 13 days after the initial stable state. (g) Comparison of the alongshore wavenumber spectra of detrended $v^{\prime}$ between the 3D (black) and 2D (red) cases. The spectra are computed over a 3-day window centered at the time when the eddy kinetic energy $K_{E}$ is maximal. (h) The cross-shore structure of basic flow in (d). The black and gray curves denote isohaline and mean flow velocity, respectively. Linear fits are carried out for isohalines from 30.1 to $31.9 \mathrm{psu}$, with an interval of $0.3 \mathrm{psu}$. Two examples of the fits (for isohalines 30.1 and 31.9 ) are shown as the black dashed lines in (h).

to zero, but the third-order momentum advection scheme we adopt has implicit numerical mixing to prevent numerical instabilities. The model solutions are not sensitive to the choices of numerical schemes applied (not shown). The main point to note in Figs. $4 a-c$ is that the coastal current is unstable. Before $t=t_{0}(=$ day 9$)$, a buoyant coastal current propagating in the $+x$ direction is stable during this developing period (Fig. 4a). Afterward, in 13 days, finite-amplitude disturbances become apparent in the surface salinity (Fig. 4b). The disturbances are particularly apparent in cross-shore velocity, which exhibits an alternating onshore/offshore wave-like pattern (Fig. 4c). The magnitude of fluctuating cross-shore velocity increasing from essentially zero to over $0.2 \mathrm{~m} \mathrm{~s}^{-1}$ in 13 days, suggesting a growing instability.
We next examine the basic flow structure and conduct companion experiments in a reentry channel. These analyses, shown in Figs. 4d-h, are to provide justifications for 1) the use of the Eady model as a building block to study baroclinic instability in coastal flows, and 2) the use of a reentry channel in the subsequent numerical experiments.

To examine the utility of simulations in a reentry channel, we construct a geostrophically balanced basic state based on a representative salinity section from the coastal current in Fig. 4a. This section is from the center of the coastal current (white vertical lines in Fig. 4a) at $t=t_{0}$ when the coastal current is stable and average over 10 adjacent alongshore grid points. We then form a uniform coastal current using this basic state and 
initialize it in a reentry channel (Fig. 4d). This setup eliminates upstream and downstream influences of bulge and nose recirculation in Fig. 4a, thus allowing a focus on local processes.

The alongshore uniform coastal current develops growing instabilities similar to those in the threedimensional (3D) setup. In Fig. 4e, disturbances are apparent along the current boundary 13 days after the initiation. Like in the three-dimensional case, the crossshore velocity shows a clear alternating onshore/offshore wave-like structure (Fig. 4f). In addition, the wavelength of the alongshore variation is similar to that of the threedimensional simulation. This is further confirmed by comparing the wavenumber spectra of the perturbation surface cross-shore velocity $v^{\prime}$ (Fig. 4g). The spectra are computed at the time of peak $\overline{v^{\prime 2}}$ bracketed by a 3-day window. The three-dimensional and alongshore uniform cases have similar spectra shapes. Both curves of spectral density, between wavelengths of 3 and $30 \mathrm{~km}$, nearly overlap and have a spectral peak at a wavelength of $25 \mathrm{~km}$ (open circles). The close correspondence suggests that the instabilities developed using an averaged basic state in a reentry channel can approximately represent those in three-dimensional coastal currents. Furthermore, diagnosing the eddy kinetic energy budget indicates the dominance of baroclinic instability, with the baroclinic energy conversion being 4 times greater than the barotropic conversion at peak $\overline{v^{\prime 2}}$ [using Eqs. (B1) and (B2) in the appendix B; not shown]. As the reentry channel permits easier diagnoses of energy and instability properties, we will adopt this approach in the subsequent experiments.

Next we examine the basic flow structure. There are general similarities shared by the coastal current and the Eady basic state. The basic flow of the coastal current has salinity surfaces that are approximately uniformly sloped and spaced (Fig. 4h). Linear fits of the isohaline slope yield a tight range of $(3.0 \pm 0.4) \times 10^{-3}$. Such salinity structure gives roughly constant $N^{2}$ and $M^{2}$ in the central region, consistent with the Eady basic state (Fig. 1a). There are however notable differences. The coastal current resides on a sloping bottom, has significant horizontal shear ( $U$ denoted by the gray contour in Fig. 4h), and is presumably subject to bottom drag due to the shallowness. Some of these factors, as discussed in section 2a, have been incorporated into the Eady model, but their combined effects have not. Nevertheless, the general similarities do provide support for the use of Eady-type models to build understanding of coastal baroclinic instability.

Below we lay out the numerical experiments that are built upon the Eady basic state. The experiments are designed to isolate the effects of nongeostrophy, boundary dissipation and bottom slope. They are carried out in a $300 \mathrm{~km}$ (alongshore) $\times 220 \mathrm{~km}$ (cross-shore) reentry channel, with uniform grid resolution of $500 \mathrm{~m}$ and 30 sigma levels. The experiments are initialized with a balanced Eady basic flow, with a constant $N^{2}$ and $M^{2}$. Random noise having a signal-to-noise ratio of 200 (i.e., velocity amplitude $\sim 1 \mathrm{~mm} \mathrm{~s}^{-1}$ ) is added to the initial basic flow to help seed the instability. The simulations then evolve freely. The parameter range is guided by a set of coastal current simulations, including that shown in Fig. 4. Table 1 summarizes three groups of experiments. Group 1 (runs 1-6) is a set to test the Eady model and nongeostrophic effects [section 2a(1)]. These simulations are inviscid and have a flat bottom (see section 3). The only controlling parameter is the Richardson number Ri [Eq. (2)], which is varied from 0.9 to 20 to cover the transition from nongeostrophic to QG regimes. Group 2 (runs 7-21) targets boundary dissipation. We hold $\mathrm{Ri}$ fixed $(\mathrm{Ri}=10$ or 15$)$ while varying the linear friction coefficient $\gamma$ to obtain different values of the friction parameter $\Delta_{E}$. The $k-\varepsilon$ turbulence closure is employed. A different bottom drag parameterization using the quadratic law was also tested (denoted by * in Table 1). Group 3 (runs 22-34) evaluates bottom slope effects. They have constant $\mathrm{Ri}$, zero drag, but various bottom slopes, with the slope parameter $\delta$ ranging from -0.75 to 0 . Dimensionally, these experiments have $H=20-40 \mathrm{~m}, N^{2}=0.09-2.56 \times 10^{-3}\left(\mathrm{~s}^{-2}\right), M^{2}=1-2 \times$ $10^{-6}\left(\mathrm{~s}^{-2}\right)$, mean flow $U=0.2-0.34\left(\mathrm{~m} \mathrm{~s}^{-1}\right)$, isopycnal slope $S=0.5-11 \times 10^{-3}$, and bottom slope $S_{B}=0.1-1 \times$ $10^{-3}$. This parameter range is consistent with that used in prior modeling studies such as Hetland (2017). Note that all experiments have a sufficiently wide $\left(\sim 10 L_{d}\right)$ baroclinic zone, and we diagnose instability properties at the center so as to minimize the influences of horizontal shear.

\section{Base case and nongeostrophic effects}

We first examine the baseline experiments (Group 1 in Table 1), with the goals of evaluating the basic properties of baroclinic instability, verifying the numerical model results, and characterizing the nongeostrophic effects. Three selected cases with $\mathrm{Ri}=15,2$, and 0.9 are shown in Fig. 5. We obtain different Ri by varying $N^{2}$ while holding $M^{2}$ and $f_{0}$ constant [see Eq. (2); $U=M^{2} H / f_{0}$ is also constant]. As $\mathrm{Ri}$ and $N^{2}$ decrease, the isopycnal slope steepens (Figs. 5a1-a3). Both the nongeostrophic effects and instability growth rate are expected to increase with decreasing $\mathrm{Ri}$ [Eq. (3)]. To make comparisons of instability structure at roughly the same stage in growth, we normalize the time by the Stone growth rate $\left(\tilde{t}=t \sigma_{\text {Stone }}\right)$. All three cases develop vigorous instabilities (Figs. 5b-d). 
TABLE 1. Summary of numerical experiments. The experiments are divided into three groups. Groups 1,2, and 3 examine the effects of nongeostrophy, boundary dissipation, and bottom slope by varying Ri [Eq. (2)], friction parameter $\Delta_{E}$ [Eq. (6)], and $\delta$ slope ratio [Eq. (10)], respectively. The terms $H$ and $U$ are the mean water depth and velocity scales; $f_{0}$ is the Coriolis parameter; $M^{2}(=-\partial \bar{b} / \partial y)$ characterizes the cross-shore buoyancy gradient of the mean flow [the overbar denotes alongshore average; buoyancy $b=-g\left(\rho-\rho_{0}\right) / \rho_{0}$; $\left.\rho_{0}=1027 \mathrm{~kg} \mathrm{~m}^{-3}\right] ; N^{2}(=\partial \bar{b} / \partial z)$ is the buoyancy frequency squared; and $S\left(=-(\partial \bar{\rho} / \partial y) /(\partial \bar{\rho} / \partial z)=M^{2} / N^{2}\right)$ is the isopycnal slope. Bottom slope $S_{B}$ is negative for depth increasing seaward. The term $\gamma$ is a linear friction factor for parameterizing the bottom stress [Eq. (4)]. Asterisks denote runs using a quadratic drag coefficient $(\mathrm{Cd})$.

\begin{tabular}{|c|c|c|c|c|c|c|c|c|c|c|c|c|}
\hline & $H(\mathrm{~m})$ & $\begin{array}{c}M^{2} \\
\left(10^{-6} \mathrm{~s}^{-2}\right)\end{array}$ & $\begin{array}{c}N^{2} \\
\left(10^{-3} \mathrm{~s}^{-2}\right)\end{array}$ & $\begin{array}{c}f_{0} \\
\left(10^{-4} \mathrm{~s}^{-1}\right)\end{array}$ & $\begin{array}{c}U \\
\left(\mathrm{~ms}^{-1}\right)\end{array}$ & $\begin{array}{c}S_{B} \\
\left(10^{-3}\right)\end{array}$ & $\begin{array}{c}S \\
\left(10^{-3}\right)\end{array}$ & $\begin{array}{c}\gamma \\
\left(10^{-4} \mathrm{~m} \mathrm{~s}^{-1}\right)\end{array}$ & $\mathrm{Ri}$ & $\Delta_{E}$ & & $\delta$ \\
\hline 1 & 20 & 1 & 0.09 & 1 & 0.2 & 0 & 11.1 & 0 & 0.9 & 0 & 0 & Group 1: varying Ri \\
\hline 2 & 20 & 1 & 0.1 & 1 & 0.2 & 0 & 10.0 & 0 & 1 & 0 & 0 & \\
\hline 3 & 20 & 1 & 0.2 & 1 & 0.2 & 0 & 5.0 & 0 & 2 & 0 & 0 & \\
\hline 4 & 20 & 1 & 0.5 & 1 & 0.2 & 0 & 2.0 & 0 & 5 & 0 & 0 & \\
\hline 5 & 20 & 1 & 1.5 & 1 & 0.2 & 0 & 0.67 & 0 & 15 & 0 & 0 & \\
\hline 6 & 20 & 1 & 2.0 & 1 & 0.2 & 0 & 0.5 & 0 & 20 & 0 & 0 & \\
\hline 7 & 20 & 1 & 1.5 & 1 & 0.2 & 0 & 0.67 & 0.5 & 15 & 0.13 & 0 & Group 2: varying \\
\hline 8 & 20 & 1 & 1.5 & I & 0.2 & 0 & 0.67 & 1.0 & 15 & 0.29 & 0 & friction parameter \\
\hline 9 & 20 & 1 & 1.5 & 1 & 0.2 & 0 & 0.67 & 2.0 & 15 & 0.59 & 0 & \\
\hline 10 & 20 & I & 1.5 & 1 & 0.2 & 0 & 0.67 & 6.0 & 15 & 1.66 & 0 & \\
\hline 11 & 20 & 1 & 1.5 & 1 & 0.2 & 0 & 0.67 & 10.0 & 15 & 2.62 & 0 & \\
\hline 12 & 20 & 2 & 2.56 & 1.25 & 0.32 & 0 & 0.78 & 0 & 10 & 0 & 0 & \\
\hline 13 & 20 & 2 & 2.56 & 1.25 & 0.32 & 0 & 0.78 & 0.5 & 10 & 0.09 & 0 & \\
\hline 14 & 20 & 2 & 2.56 & 1.25 & 0.32 & 0 & 0.78 & 1.0 & 10 & 0.17 & 0 & \\
\hline 15 & 20 & 2 & 2.56 & 1.25 & 0.32 & 0 & 0.78 & 2.0 & 10 & 0.36 & 0 & \\
\hline 16 & 20 & 2 & 2.56 & 1.25 & 0.32 & 0 & 0.78 & 6.0 & 10 & 1.01 & 0 & \\
\hline 17 & 20 & 2 & 2.56 & 1.25 & 0.32 & 0 & 0.78 & 10.0 & 10 & 1.68 & 0 & \\
\hline 18 & 20 & 2 & 2.56 & 1.25 & 0.32 & 0 & 0.78 & $10^{*}$ & 10 & 0.57 & 0 & \\
\hline 19 & 20 & 2 & 2.56 & 1.25 & 0.32 & 0 & 0.78 & $20 *$ & 10 & 1.14 & 0 & \\
\hline 20 & 20 & 2 & 2.56 & 1.25 & 0.32 & 0 & 0.78 & $50 *$ & 10 & 2.80 & 0 & \\
\hline 21 & 20 & 2 & 2.56 & 1.25 & 0.32 & 0 & 0.78 & $100^{*}$ & 10 & 5.48 & 0 & \\
\hline 22 & 40 & 1 & 0.5 & 1.40 & 0.29 & 0 & 2.0 & 0 & 10 & 0 & 0 & Group 3: varying slope \\
\hline 23 & 40 & 1 & 0.5 & 1.40 & 0.29 & -0.2 & 2.0 & 0 & 10 & 0 & -0.1 & \\
\hline 24 & 40 & 1 & 0.5 & 1.40 & 0.29 & -0.4 & 2.0 & 0 & 10 & 0 & -0.2 & \\
\hline 25 & 40 & 1 & 0.5 & 1.40 & 0.29 & -0.5 & 2.0 & 0 & 10 & 0 & -0.26 & \\
\hline 26 & 40 & I & 0.5 & 1.40 & 0.29 & -0.6 & 2.0 & 0 & 10 & 0 & -0.31 & \\
\hline 27 & 40 & I & 0.5 & 1.40 & 0.29 & -0.8 & 2.0 & 0 & 10 & 0 & -0.41 & \\
\hline 28 & 20 & 1 & 1.5 & 1 & 0.2 & -0.1 & 0.67 & 0 & 15 & 0 & -0.15 & \\
\hline 29 & 20 & 1 & 1.5 & 1 & 0.2 & -0.2 & 0.67 & 0 & 15 & 0 & -0.3 & \\
\hline 30 & 20 & 1 & 1.5 & 1 & 0.2 & -0.5 & 0.67 & 0 & 15 & 0 & -0.75 & \\
\hline 31 & 40 & 1.2 & 0.73 & 1.4 & 0.34 & 0 & 1.4 & 0 & 10 & 0 & 0 & \\
\hline 32 & 40 & 1.2 & 0.73 & 1.4 & 0.34 & -0.5 & 1.4 & 0 & 10 & 0 & -0.31 & \\
\hline 33 & 40 & 1.2 & 0.73 & 1.4 & 0.34 & -0.8 & 1.4 & 0 & 10 & 0 & -0.49 & \\
\hline 34 & 40 & 1.2 & 0.73 & 1.4 & 0.34 & -1.0 & 1.4 & 0 & 10 & 0 & -0.61 & \\
\hline
\end{tabular}

The case with a smaller Ri becomes unstable earlier, consistent with the expectation of $\sigma \propto(1+\mathrm{Ri})^{-1 / 2}$ in Eq. (3). For example, with $\mathrm{Ri}=15$, the wave-like disturbances are not apparent until around day 18 (Fig. 5c1). But, for $\mathrm{Ri}=2$, disturbances are already visible at day 8 . The spatial scale of the instability is also shorter with smaller $\mathrm{Ri}$, because of the shorter deformation radius (i.e., smaller $L_{d}$ for smaller $N^{2}$ ).

The growing disturbances are driven by baroclinic instability. Time series in Fig. 5e clearly show the sharp growth of cross-shore velocity variance $\overline{v^{\prime 2}}$ (solid curve; overbar is alongshore mean; prime is perturbation). The variance is computed at each cross-shore location and then averaged across the center half of the mean flow width to obtain a representative time series [i.e., width $(\sim 75 \mathrm{~km}$ here $)$ is defined as the cross-shore span over which $N^{2}$ and $M^{2}$ are vertically uniform; see Fig. 5a1]. The standard deviation of the width average is negligibly small. To diagnose the energy source of growing instability, we evaluate the volume integrated eddy kinetic energy $K_{E}$ budget [Eqs. (B1) and (B2) in appendix B]. Because the domain is a reentry channel and the problem is inviscid, energy flux divergences vanish after integrating over the entire domain, and dissipation is zero by definition. This leaves the $K_{E}$ tendency to be balanced by baroclinic and barotropic energy conversions [Eq. (B2)]. The tendency (black curves) is completely governed by baroclinic energy conversion (red curves) 

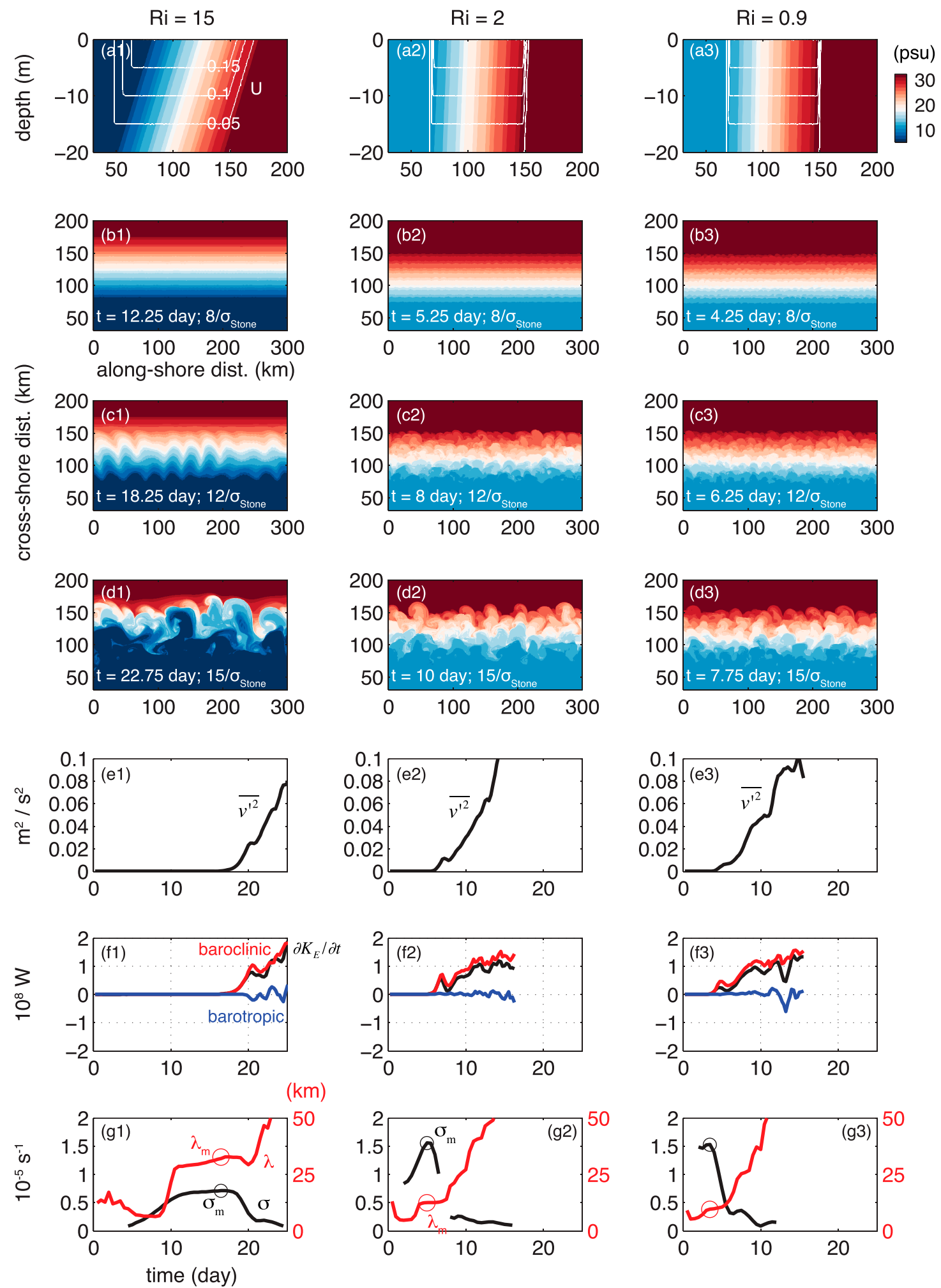

FIG. 5. Comparisons of instability properties among cases with different $\mathrm{Ri}$. The cases from left to right have $\mathrm{Ri}=15,2$, and 0.9 (cases 1, 3, and 5 in Table 1). Their basic flows have the same $M^{2}, f_{0}, H$, and $U$, but different $N^{2}$. (a) The cross-shore basic flow and salinity structure. (b)-(d) The top view of salinity taken at $\tilde{t}=t \sigma_{\text {Stone }}=8,12$, and 15 [i.e., time normalized by the Stone growth rate in Eq. (3)]. (e),(f) The time series of $\overline{v^{2}}$ and the $K_{E}$ budget [Eq. (B2)]. (g) The time series of estimated growth rate (black) and energy-weighted wavelength (red). The most unstable mode ( $\sigma_{m}$ and $\lambda_{m}$; denoted by open circles) is taken at the maximum growth rate. 
in all cases, indicating the dominance of baroclinic instability (Fig. 5f). Contributions of barotropic conversion (blue curves) are negligible as the basic flows have zero horizontal shear except near the edges. Note that $\overline{v^{2}}$ and the $K_{E}$ budget in Fig. 5 are only evaluated before the instability reaches the channel walls to avoid boundary influences.

Next we quantify the growth rate and dominant wavelength of the instability. For growth rate, we fit the time series of $\overline{v^{2}}$ to an exponential curve over a 3-day moving window (e.g., Brink 2012). The estimated growth rate, denoted by the black curves in Fig. 5g, shows large time variations. It has a peak early in time when the instability is of small amplitude and the energy level is low (e.g., near day 5 in Figs. 5g2,e2). The growth rate then drops sharply when the energy level increases (e.g., after day 10 in Fig. 5 g2), implying saturation due to nonlinear interactions (e.g., Fox-Kemper et al. 2008; Radko et al. 2014). Because our objective is to examine the utility of linear Eady-type theories, we focus on the early stage of instability and take the peak growth rate to represent the most unstable mode $\left(\sigma_{m}\right.$; black circle in Fig. $5 \mathrm{~g}$ ). It can be seen that $\sigma_{m}$ for $\mathrm{Ri}=2$ is greater than that for $\mathrm{Ri}=15$, as expected from Eady/Stone theories (see below). The growth rate estimates are not sensitive to the moving window size that was varied between 2 and 5 days. Using $K_{E}$ time series yield nearly identical results as $\overline{v^{\prime 2}}$.

To determine the dominant wavelength, we compute the spectra of surface $v^{\prime}$ across the mean flow and obtain the wavenumber of the spectral peak at each $y$ location. The wavenumbers are then weighted by the values of spectral peak to obtain an energy-weighted mean wavenumber $k$ (Spall 2007; Brink 2012). The time series of the dominant wavelength is shown as the red curve in Fig. 5g. Again we take the wavelength at the maximal growth rate to represent the most unstable wave mode $\lambda_{m}$ (red circles). It can be seen that the wavelength is relatively steady around $\lambda_{m}$, suggesting that our estimate is reasonably robust. This relatively steady period is also consistent with the linear stage of instability when nonlinear cross-scale energy transfer is negligible. Afterward, the wavelength increases sharply while the growth rate drops. This is indicative of nonlinear inverse energy cascade that shifts the dominant mode to larger scales.

Overall, the model-derived dependences of most unstable growth rate and wavelength on $\mathrm{Ri}$ agree reasonably well with the Stone predictions (Fig. 6). For $\mathrm{Ri}>5$ where neglecting nongeostrophic effects only amounts to a less than $10 \%$ error and the Eady theory is valid, the model results and the Stone and Eady theories collapse. But, as Ri decreases, nongeostrophic effects become
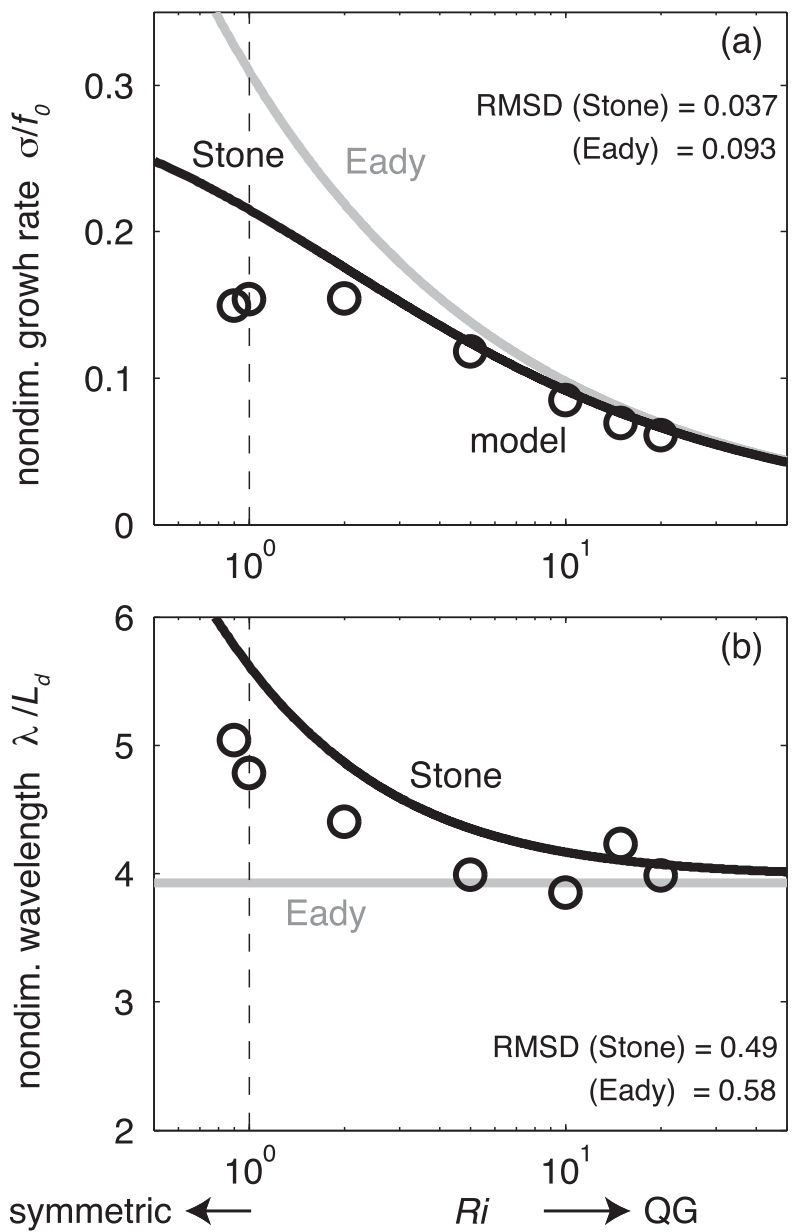

FIG. 6. (a) The most unstable growth rate and (b) wavelength as a function of Ri. The circles are model-derived $\sigma_{m}$ and $\lambda_{m}$. Black and gray curves are the Stone and Eady predictions [Eqs. (3) and (1)]. The QG regime is toward the right (e.g., $\mathrm{Ri}=\mathrm{Ro}^{-2}$ ), whereas the symmetric instability can occur when $\mathrm{Ri}<1$. The RMSDs between model-derived values and theoretical predictions) are given.

increasingly more important, and the Stone theory gives a better estimate of growth rate and wavelength. This is reflected in that the root-mean-square deviation (RMSD) of the Stone solution is smaller than Eady by a factor of 2.5 and 1.2 for growth rate and wavelength, respectively. For $\mathrm{Ri}<1$ (indicated by the dashed line), $\sigma_{m}$ deviates from the theories due mainly to the influences of symmetric instability. For a basic flow without horizontal shear, $\mathrm{Ri}<1$ corresponds to a negative Ertel potential vorticity [e.g., see Eq. (11) in Thomas et al. 2008], permitting symmetric instability to occur. Indeed, from the simulation of case $1(\mathrm{Ri}=0.9)$, we see rolls of vertical motion in the $y-z$ plane that are generally along isopycnal surfaces (not shown), consistent with the slant convection triggered by symmetric instability. However, this slantwise motion with a fine 
cross-shore scale $(\sim 2-3 \mathrm{~km})$ is only short lived (less than a day) and is soon swamped by more cross-shore uniform motion of baroclinic instability. The interplay of baroclinic and symmetric instabilities is beyond the scope of this study and thus not explored further.

The growth rate $\left(\sigma_{m} / f_{0}\right)$ increases with decreasing $\mathrm{Ri}$ in the regime dominated by baroclinic instability (Fig. 6; $\mathrm{Ri}>1$ ). This is because steepening of isopycnals with decreasing $\mathrm{Ri}$ allows the disturbances to extract more potential energy (as discussed in section 2a). Moreover, when compared with the Eady mode, the nongeostrophic baroclinic instability has a lower growth rate and a longer wavelength. Nakamura (1988) explained this shift toward a longer mode by the shallowing of boundary-trapped waves as the nongeostrophic effect increases. The analyses in the appendix B support Nakamura's explanation (see below).

In addition to the growth rate and wavelength, the baroclinic instability in these cases exhibits other wave characteristics that agree with the classic theories. The evidence is described in appendix B. Briefly, we estimate the wave speed $c$ and phase via cross correlations. Vertical structure of the instability is diagnosed with the root-mean-square of $v^{\prime}\left(v_{\mathrm{rms}}^{\prime}\right)$. The results shown by Fig. B1 provide support for 1) phase locking of counterpropagating, boundary-trapped waves, with $c=U / 2$ for all cases; 2) westward phase tilt with top-bottom phase difference near $\pi / 2$; and 3) instability (wave) amplitude that agrees with Eady solution in QG regime (large Ri) but becomes shallower (i.e., faster decay away from the boundary) in nongeostrophic cases as explained by Nakamura (1988).

Overall, the consistency between these cases (varying $\mathrm{Ri}$, inviscid, flat bottom) and the Eady/Stone theories suggests that the Eady-type numerical experiments provide a good test bed for studying baroclinic instability in coastal flows. It also lends support to the use of the analysis, with quantifications of growth rate, wavelength, phase speed, and $K_{E}$ budget, to further explore how the instability is affected by boundary dissipation and bottom slope.

\section{Influences of boundary dissipation}

\section{a. Structure and growth rate}

We study the effects of bottom boundary dissipation via examining the experiment Group 2 (run 7-21 in Table 1). Like Fig. 5, Fig. 7 compares the spatial structure of instability, $K_{E}$, and growth rate/wavelength among three cases (run 12,14, 17). These cases have identical basic flow, with $\mathrm{Ri}=10$. They only differ in the linear friction coefficients used $\left(\gamma=0,10^{-4}, 10^{-3} \mathrm{~m} \mathrm{~s}^{-1}\right)$, and that the viscous cases have $k-\varepsilon$ closure turned on.
The friction factors correspond to $\Delta_{E}$ of $0,0.17$, and 1.7 [Eq. (6)] or Ekman number $E_{k}\left[=\gamma /\left(f_{0} H\right)\right]$ of $0,0.04$, and 0.4 . The linear and quadratic friction coefficients tested here span a realistic range of values for coastal flows $\left[\gamma=10^{-5} \sim 10^{-3}\left(\mathrm{~m} \mathrm{~s}^{-1}\right)\right.$ as in Brink and Cherian (2013); $\left.\mathrm{Cd}=10^{-3} \sim 10^{-2}\right]$.

Several conclusions can be drawn from this analysis. First, instabilities occur in all cases, as the disturbances show clear amplification in less than 15 days (Fig. 7c). Including bottom drag reduces the energy level. For example, the maximum value of $\overline{v^{2}}$ in Fig. 7d decreases with increasing $\Delta_{E}$. However, a friction coefficient as large as $10^{-3} \mathrm{~m} \mathrm{~s}^{-1}$ does not stabilize the flow.

Second, from the $K_{E}$ budget in Figs. 7e2 and 7e3, baroclinic instability is responsible for the disturbance growth, as the baroclinic energy conversion (red curve) dominates the initial increase of $K_{E}$. Barotropic instability is again unimportant. After the initial growth, a steady balance between baroclinic conversion and dissipation is reached and maintained over an extended period.

Third, unlike the inviscid case (Fig. 7f1), the time series of growth rate in the viscous cases (Figs. 7f2,f3) show two peaks. Diagnosing the Ertel PV suggests that the first and second peaks correspond to symmetric and baroclinic instabilities, respectively. In the viscous cases, symmetric instability occurs near the surface in the beginning, as shown by the finescale wiggles in Figs. 7a2 and 7a3. This is because the no-flux boundary condition forces the near-boundary isopycnals to steepen such that local PV becomes negative. However, the symmetric instability is short lived as the surface density adjusts rapidly to a symmetrically stable state. This instability has no appreciable impact on the mean density structure (cf. Figs. 7a1,a2), consistent with the findings of Brink and Cherian (2013). After the initial adjustment, baroclinic instability (second peak), which requires sustained and organized wave interactions, begins to dominate (e.g., Haine and Marshall 1998). The second peak in growth rate also corresponds to the sharp rise in baroclinic conversion (cf. Figs. 7e2-f2), suggesting the onset of baroclinic instability. Since our focus is on baroclinic instability, we take the second peak in growth rate and the corresponding wavelength (black and red squares) to represent the most unstable mode.

\section{b. Testing the Eady-Ekman theory}

One main objective of this study is to evaluate whether the Eady-Ekman theory can provide guidance in understanding how coastal baroclinic instability responds to varying bottom drag and dissipation. To evaluate the theory, we plot the model-derived growth rate and wavelength against the friction parameter $\Delta_{E}$ for different values of Ri (Figs. 8a,c). The inviscid Eady 

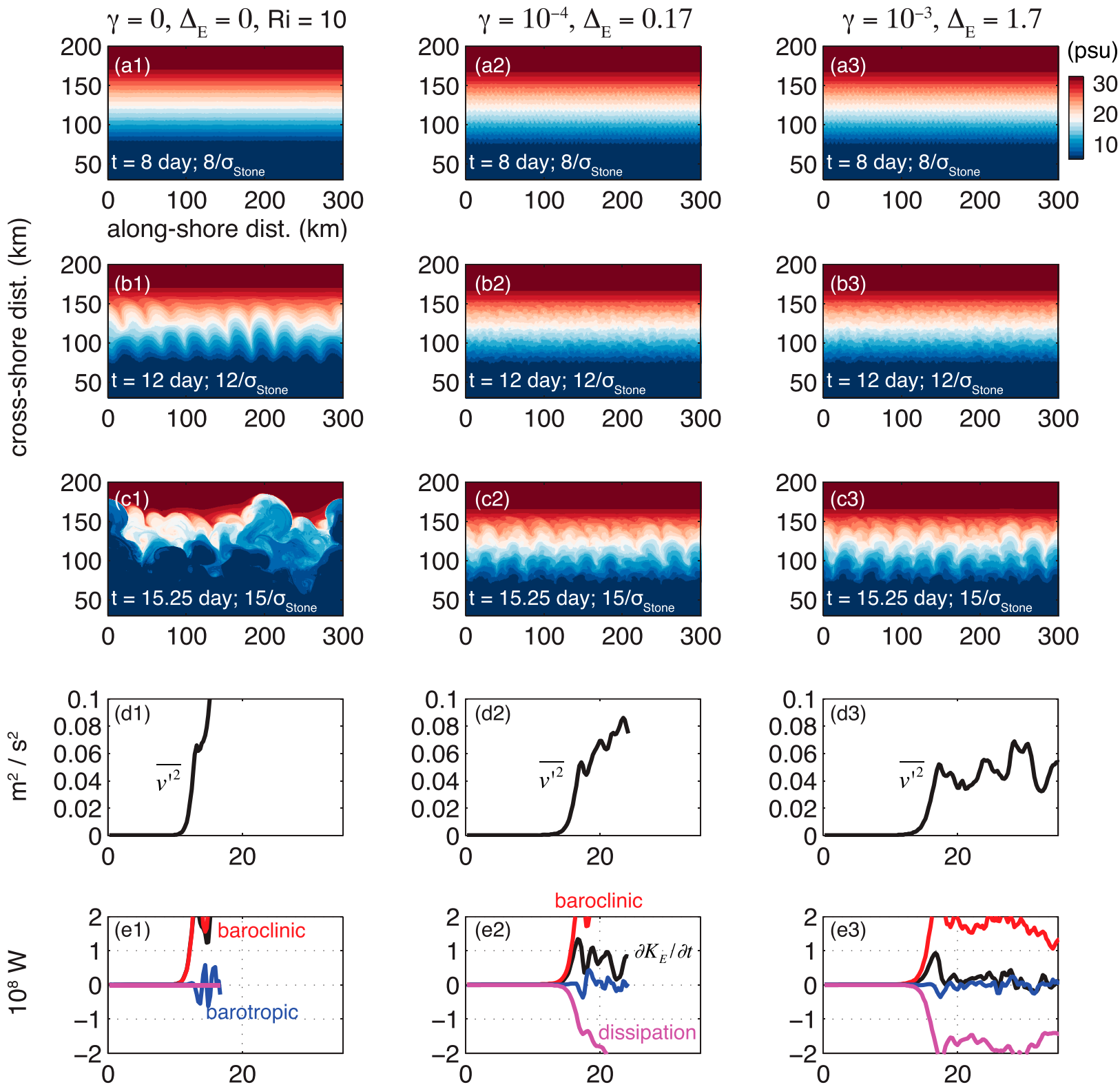

$(\mathrm{km})$
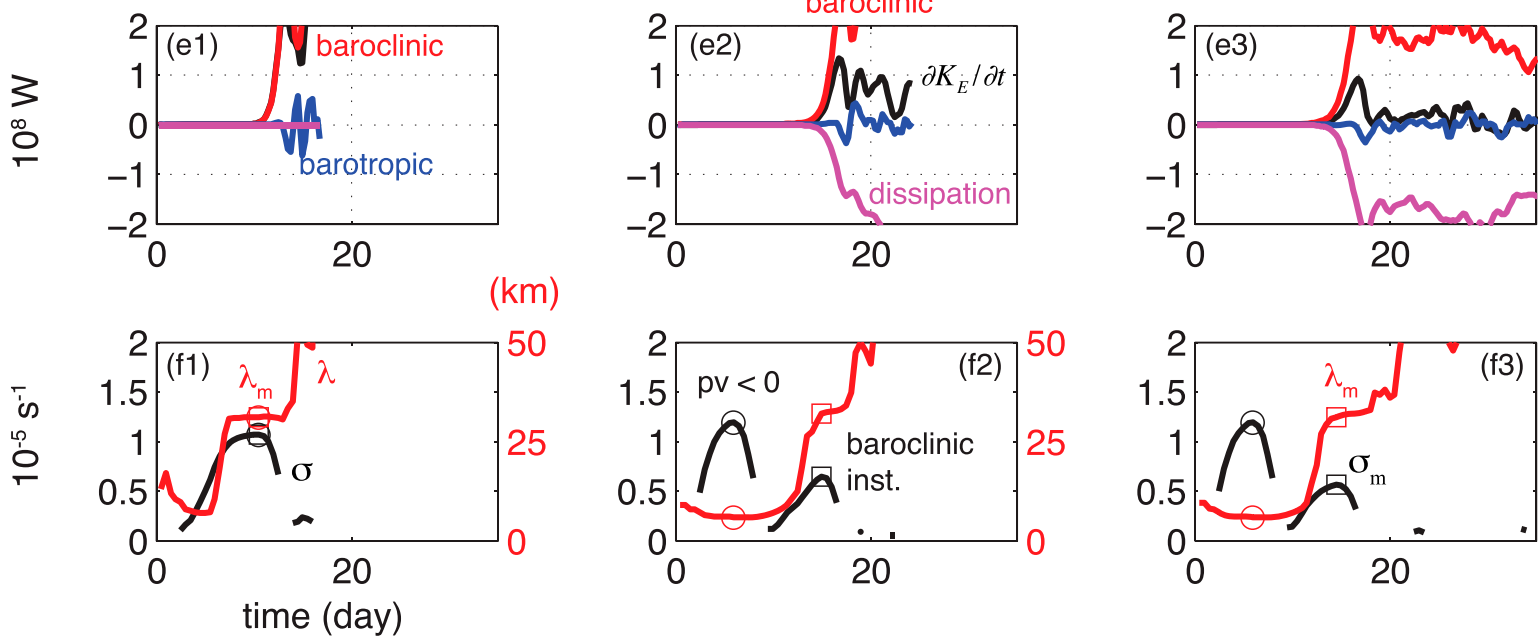

FIG. 7. As in Fig. 5, but here Ri is held fixed $(=10)$. The three cases differ only in the linear friction factor used $\left(\gamma=0,10^{-4}\right.$, and $10^{-3} \mathrm{~m} \mathrm{~s}^{-1}$; cases 12,14, and 17 in Table 1), corresponding to $\Delta_{E}=0,0.17$, and 1.7. Note that the basic flows (i.e., Fig. 5a) are not plotted because they are identical. 
Original Eady Ekman
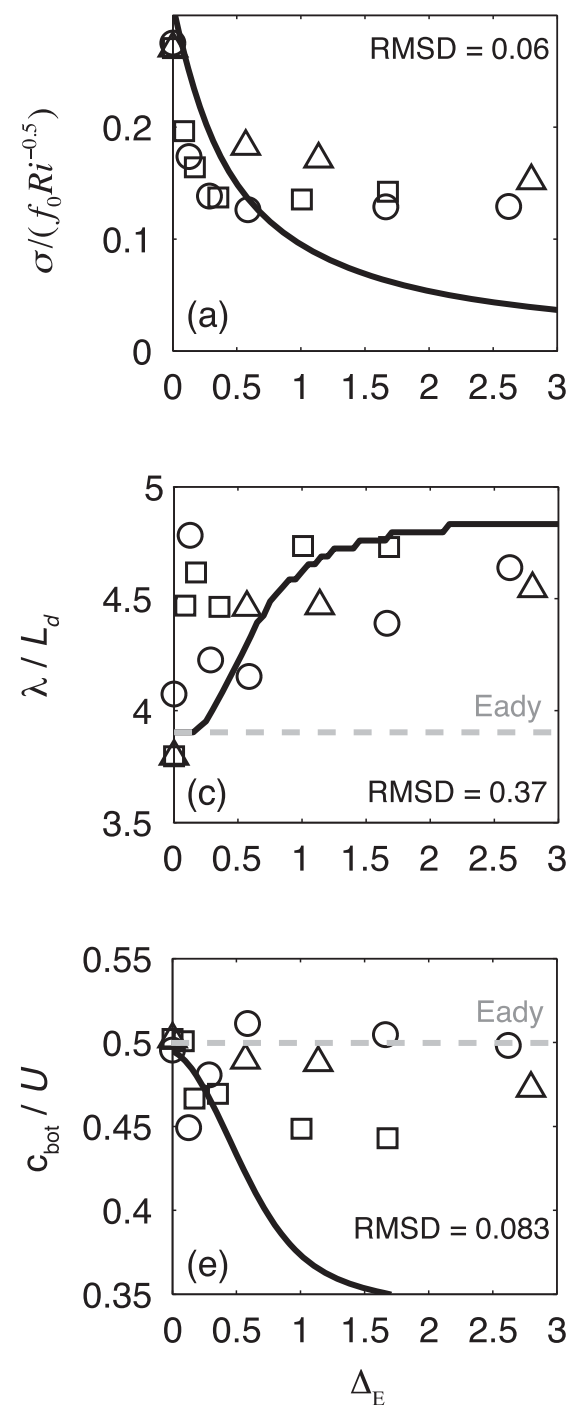

With pumping correction
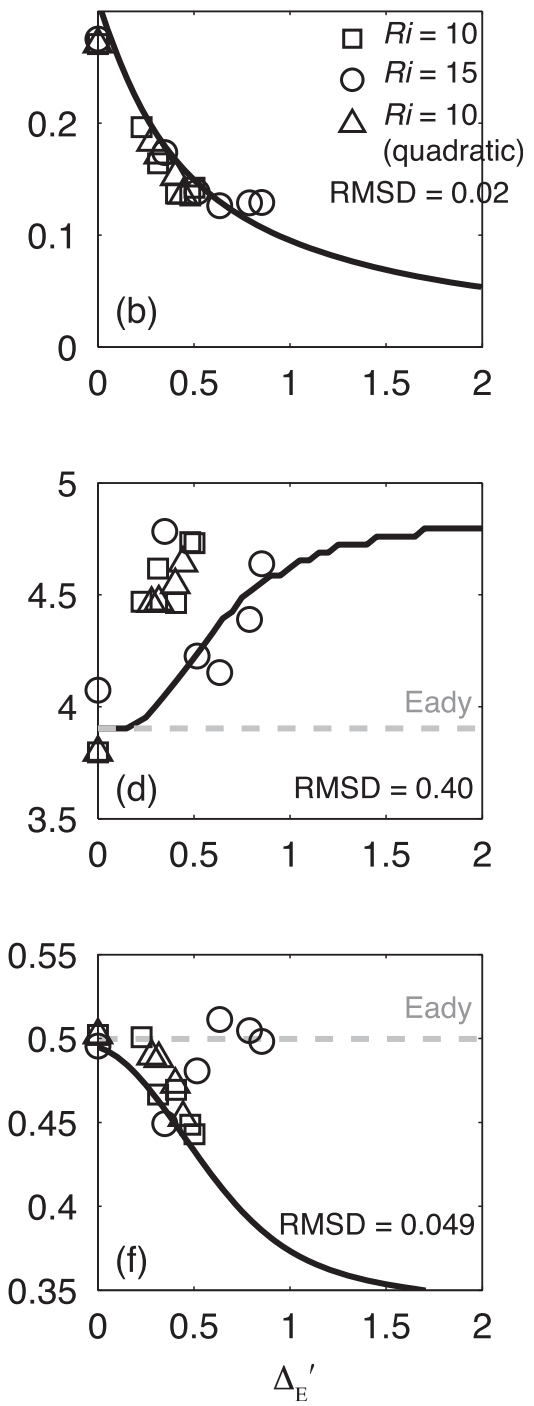

FIG. 8. Evaluation of the Eady-Ekman theory. (a),(b) Growth rate, (c),(d) wavelength, and (e),(f) bottom wave speed are plotted against the friction parameter $\Delta_{E}$. These quantities are nondimensionalized by $f_{0} \mathrm{Ri}^{-0.5}, L_{d}$, and $U$, respectively [scales guided by the Eady model; Eq. (1)]. (left) The original theory, with $\Delta_{E}$ defined in Eq. (6). (right) Shown with $\Delta_{E}^{\prime}$ [Eq. (13)] that includes a correction for Ekman pumping at large drag. The model-derived quantities are the open symbols. Theory is the black curve. The horizontal dashed lines indicate the Eady solution. Wave speed estimates are described in appendix B. The RMSDs for the model-theory comparisons are given.

solution is at $\Delta_{E}=0$ [Eq. (1)]. Experiments using a quadratic drag law are also included.

The Eady-Ekman theory captures the general respons of baroclinic instability to increasing frictional effects: The growth rate decreases, while the instability shifts toward longer wavelength, presumably due to the weakening of bottom buoyancy perturbations that slow the bottom waves (Fig. 8e; see appendix B for how wave phase speed is estimated), forcing the instability to select a faster and longer wave mode to maintain the coupling (e.g., Fig. 2b, section 2a; see more below). However, the theory overestimates the reduction of growth rate at large drag. For $\Delta_{E}>0.5$ the modelderived growth rate levels off, suggesting that the baroclinic instability becomes insensitive to further increases in friction coefficient. Yet, the theory predicts a continued growth rate reduction. At $\Delta_{E}=2.8$, for example, the reduction is overestimated by the theory by 
more than $70 \%$. The wavelength and bottom wave speed comparisons in Figs. $8 \mathrm{c}$ and 8e also exhibit a large degree of scatter.

To further evaluate the validity of the theory and to look into the causes for the discrepancies at large drag, we examine the assumptions of the Eady-Ekman model. As schematized in Fig. 2a, the key assumptions are 1) vertical segregation of inviscid interior and a boundary layer (the instability grows in the inviscid interior, while dissipation is concentrated in a thin boundary layer) and 2) interior instability is forced from below by Ekman pumping (bottom stress induces Ekman transport). The transport divergence then drives Ekman pumping that modifies the interior instability.

Assuming separation of an energy converting interior and a dissipative boundary layer is supported by the model results in all cases. As an example, we show the cross-shore structure of baroclinic energy conversion and dissipation diagnosed from the $K_{E}$ budget [Eq. (B1)] for a simulation with large drag $\left(\gamma=10^{-3} \mathrm{~m} \mathrm{~s}^{-1}\right.$; $\Delta_{E}=1.7$; Fig. 9). Both processes occur quite uniformly across the mean flow center, and are clearly segregated in the vertical. The energy conversion takes place above a thin boundary layer as denoted by the horizontal line, whereas most of the energy dissipation occurs near the bottom. Here we use the height where $v_{\text {rms }}^{\prime}$ has a subsurface maximum to define the boundary layer (see below). The region above this height accounts for over $95 \%$ of the energy conversion, whereas over $80 \%$ of the dissipation is found below. Similar patterns hold for all cases.

Next we examine the vertical structure of the instability and bottom Ekman flows for different values of $\Delta_{E}$ (Fig. 10). The top panels show the vertical structure of $v_{\mathrm{rms}}^{\prime}$ at maximal $\sigma_{m}$, averaged across the mean flow center (over $1 L_{d}$ ). The presence of bottom drag greatly reduces the near-bottom velocity, compared to the Eady model, such that $v_{\text {rms }}^{\prime}$ exhibits a subsurface secondary maximum. The height of the $v_{\text {rms }}^{\prime}$ secondary maximum (dashed lines) represents, on average, the zero-crossing of stress profiles and is thus used to set the boundary layer thickness $d_{v^{\prime} \max }$. Above $d_{v^{\prime} \max }$, the amplitude of instability increases toward both boundaries like in the Eady model. However, unlike the symmetric Eady structure, $v_{\text {rms }}^{\prime}$ with bottom drag is surface intensified. This surface intensification is consistent with Fig. 2a and Eq. (7). Ekman pumping acts to reduce the bottom buoyancy perturbations, weakening the bottom waves (see below).

The bottom Ekman transport is a robust feature in all cases. In Fig. 10b, we compute the alongshore component of the transport $\left(-\int u-u_{g} d z\right)$ and plot it against the predictions of $\tau_{b y}^{\prime} /\left(\rho_{0} f_{0}\right)$ over the entire domain. The transport is integrated from the bottom to $d_{v^{\prime} \max }$. The

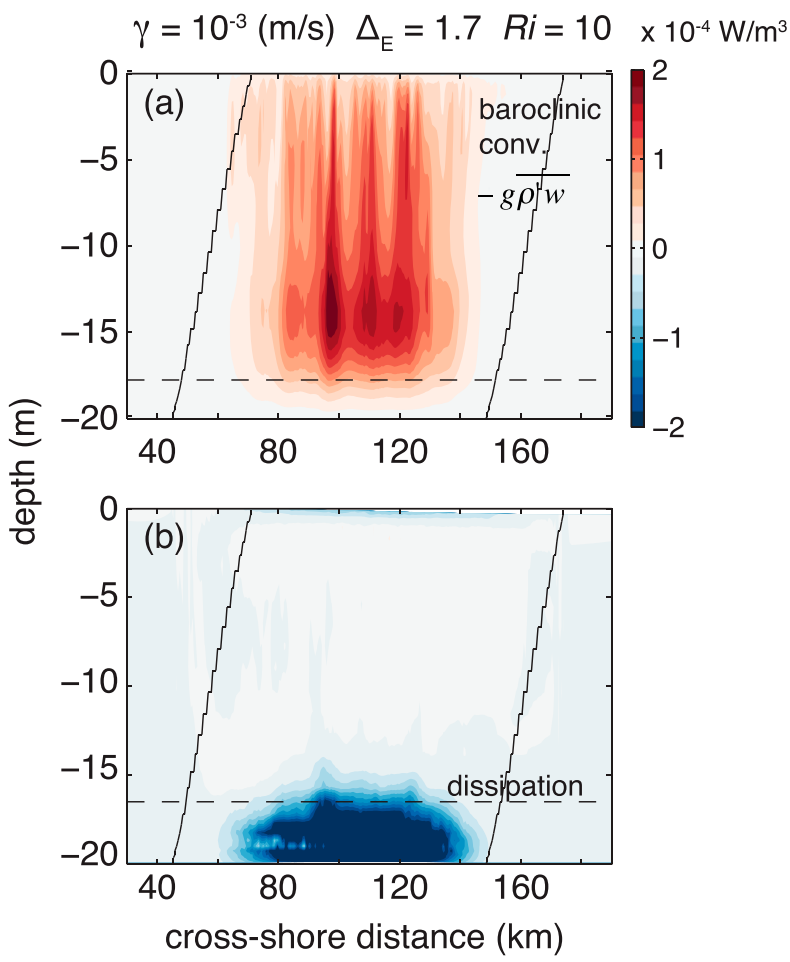

FIG. 9. An evaluation of the conceptual Eady-Ekman model that assumes vertical separation of an inviscid interior and a dissipative boundary layer (see Fig. 2a).The cross-shore structure of (a) baroclinic energy conversion and (b) energy dissipation [see Eq. (B1) in appendix $\mathrm{B}]$. We choose the case with a largest friction factor as an example (case 17; Fig. 7a3). The structure is taken at maximal growth rate (square symbol in Fig. 7f3). Two sloped lines indicate the surface salinity minimum and maximum that define the baroclinic zone (e.g., Fig. 5a1). The horizontal dashed lines denote the bottom boundary layer height (see Fig. 10a and corresponding text).

comparisons mostly follow the one-to-one relationship, suggesting the dominance of Ekman flows in the bottom layer. Note that, for the case with large drag (Fig. 10b3), we integrate the transport to a height $1 \mathrm{~m}$ above $d_{v^{\prime} \max }$ because $d_{v^{\prime} \max }$ slightly underestimates the boundary layer thickness.

While the above analyses support the assumptions of inviscid interior and bottom Ekman flows, the Ekman pumping formulation in the Eady-Ekman theory is found to be problematic at large drag. The pumping velocity at the top of a bottom Ekman layer $[w(z=d)]$ is proportional to the curl of bottom stress [Eq. (4)]. If we parameterize the bottom stress using the interior velocity at $z=d_{v^{\prime} \text { max }}$, in the case of linear friction, the vertical velocity can be written in terms of interior vorticity $w=\left(\gamma / f_{0}\right) \zeta_{v^{\prime} \max }$ as in the Eady-Ekman theory. However, our numerical simulations resolve the boundary layer such that the bottom stress is computed using the velocity at the lowest grid point (Warner et al. 2008; Taylor and Sarkar 2008). This means that, for 

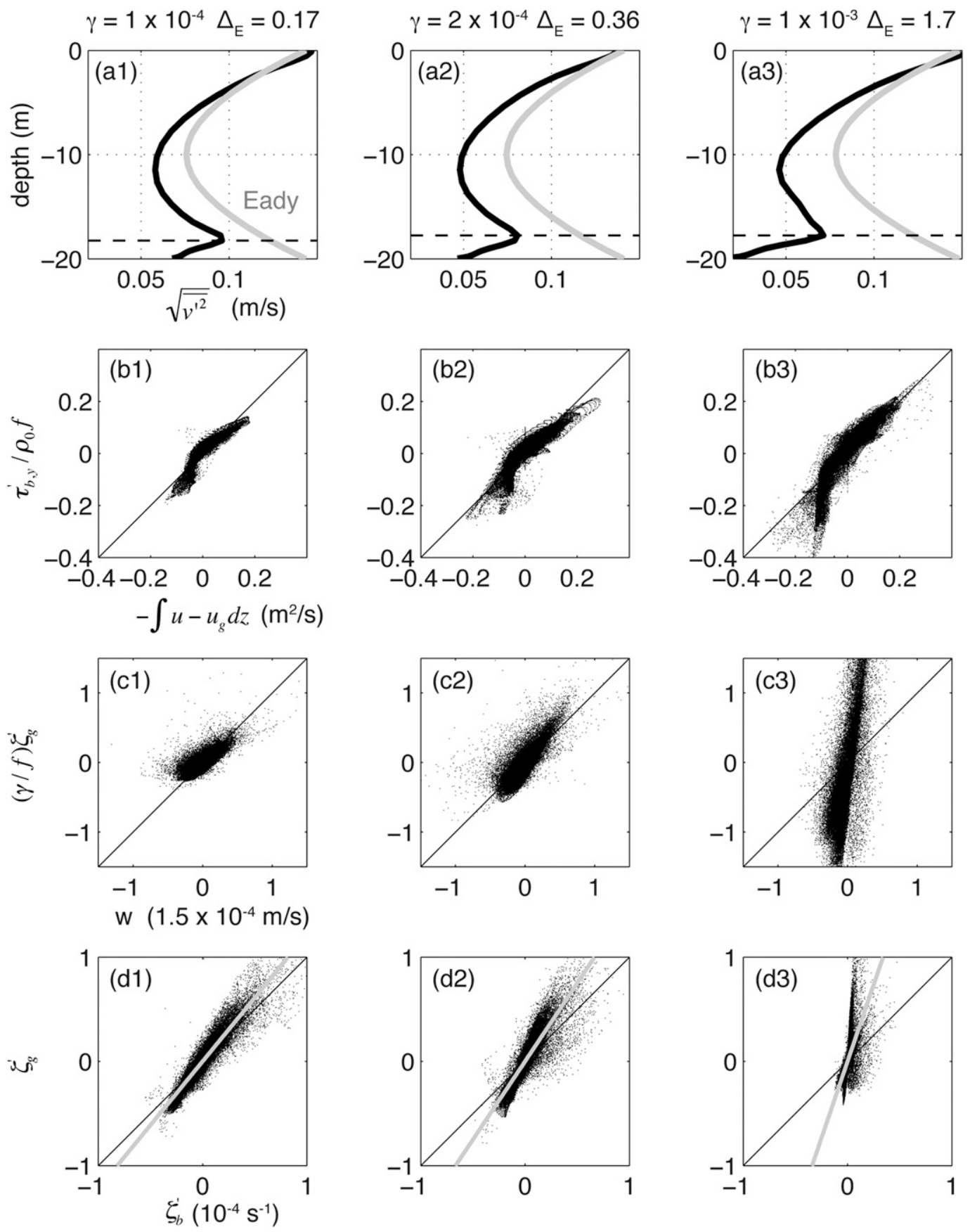

FIG. 10. Comparisons of (a) the vertical structure of unstable wave amplitude $\left(v_{\mathrm{rms}}^{\prime}=\sqrt{\overline{v^{2}}}\right)$, (b) alongshore component of Ekman transport, (c) Ekman pumping formulation [Eq. (4)], and (d) bottom vorticity representation, for three frictional cases $\left(\Delta_{E}=0.17,0.36\right.$, and 1.7 from left to right). In (a), the horizontal dashed line denotes the subsurface maximum of $v_{\text {rms }}^{\prime}\left(d_{v^{\prime} \max }\right)$ that roughly represents the height of zero stress and thus boundary layer limit. The structure of Eady wave is the gray curve. In (d) bottom vorticity $\zeta_{b}$ vs interior vorticity $\zeta_{g}$ is shown. The EadyEkman theory assumes that $\zeta_{b}$ can be approximated by $\zeta_{g}$ (see text). In (b), (c), and (d), the geostrophic velocity $u_{g}$, vertical velocity $w$, and $\zeta_{g}$ are taken immediately above the boundary layer. The black dots are the model results over the entire domain. Note that for the large drag case in (b3), because using $d_{v^{\prime} \max }$ tends to underestimate the boundary layer height, we compute the Ekman transport at $1 \mathrm{~m}$ above $d_{v^{\prime} \text { max }}$. In (d), the gray lines are the linear fits, with slopes of $1.2,1.5$, and 2.9 and a $R^{2}$ of $0.9,0.8$, and 0.4 , respectively. 
linear friction, the correct representation of Ekman pumping is $w=\left(\gamma / f_{0}\right) \zeta_{b}$, where $\zeta_{b}$ is the near-bottom vorticity. Hence, the use of the Ekman pumping formulation in the theory [Eq. (4)] is equivalent to assuming that the near-bottom vorticity $\zeta_{b}$ can be approximated by the interior value $\zeta_{v^{\prime} \max }$.

The assumption of $\zeta_{b} \approx \zeta_{v^{\prime} \max }$ is rather poor at large drag. For relatively small drag $\left(\gamma \leq 2 \times 10^{-4} \mathrm{~m} \mathrm{~s}^{-1}\right)$, the model-derived vertical velocity $w$ at the top of boundary layer $\left(z=d_{v^{\prime} \max }\right)$ is consistent with the Ekman pumping predictions evaluated at the same height (Figs. 10c1,c2). The interior vorticity is slightly greater than the nearbottom value, but they generally follow the one-to-one relationship (Figs. 10d1,d2). For example, in Fig. 10d1, a linear regression gives $\zeta_{v^{\prime} \max } \approx 1.2 \zeta_{b}$, meaning that using the interior vorticity to approximate $\zeta_{b}$ leads to a $20 \%$ error. By contrast, at large drag in Fig. 10c3, the Ekman pumping predictions greatly overestimate the vertical velocity. The regression in Fig. 10d 3 suggests that the use of $\zeta_{v^{\prime} \max }$ overpredicts $\zeta_{b}$ by a factor of 3 . Such symptoms also carry over to the cases with large quadratic drag coefficients (runs 20-21 in Table 1). As a result, the overestimated Ekman pumping in the theory provides too large of a boundary forcing to the interior or too much reduction in buoyancy perturbations [Fig. 2a and Eq. (7)], which in turn leads to an overestimation of growth rate reduction at large drag in Fig. 8a.

\section{c. Influences of a stratified Ekman layer}

The overestimation of near-bottom vorticity and thus Ekman pumping using interior vorticity is apparent in the velocity profiles. The difference in $v_{\mathrm{rms}}^{\prime}$ across the boundary layer in Fig. 10a3 (large drag) is clearly larger than that in Fig. 10a1 (small drag). To investigate what causes the velocity deficit to increase, we examine the near-bottom structure of the flow (Fig. 11).

The observed increase in top-bottom velocity difference with increasing drag is consistent with the response of a stratified Ekman layer. The deficit of $v_{\mathrm{rms}}^{\prime}$ is quite small $\left(\sim 1 \mathrm{~cm} \mathrm{~s}^{-1}\right)$ with $\gamma=5 \times 10^{-5}$ (gray curve; Fig. 11b), but it increases to over $5 \mathrm{~cm} \mathrm{~s}^{-1}$ as $\gamma$ increases to $10^{-3}$ (black curve). Although the boundary layers are thin $(<3 \mathrm{~m})$, they remain stratified from about $0.5 \mathrm{~m}$ above the bottom, and their maximal $N^{2}$ is not far from the basic flow value of $2.56 \times 10^{-3} \mathrm{~s}^{-2}$ (Fig. 11a). The vertical shear $\sqrt{(\partial u / \partial z)^{2}+(\partial v / \partial z)^{2}}$ generally increases toward the bottom, but it has a notable subsurface maximum (Fig. 11c). Here we overlay a scaling theory proposed by Taylor and Sarkar (2008) for stratified Ekman layer (dashed curves). The scaling relates the shear with the classic law of the wall and overlying stratification as

$$
\frac{d V}{d z_{\mathrm{ab}}} \approx \frac{u_{*}}{\kappa z_{\mathrm{ab}}}+N
$$

where $u_{*}\left(=\sqrt{\tau_{b}^{\prime} / \rho_{0}}\right)$ is the friction velocity, $\kappa$ is the von Kármán constant, and $z_{\mathrm{ab}}$ is height above bottom. Without coefficient tuning, the scaling of shear for a stratified Ekman layer shows vertical structure similar to the model results (Fig. 11c). It suggests that the downward increase of shear corresponds to the structure of logarithmic velocity profile, but the shear of log law decays rapidly as $1 / z_{\mathrm{ab}}$. Away from the bottom, the shear is supported by interior stratification $N$, thereby showing a subsurface maximum. Indeed, the shear at the near-bottom grid scales linearly with the log law $u_{*} /\left(\kappa z_{\mathrm{ab}}\right)$ (Fig. 11d; squares), whereas the shear over the upper half of $d_{v^{\prime} \max }$ is proportional to stratification $N$ (circles).

\section{d. An empirical correction for the Ekman pumping}

The above analyses have shown that the Eady-Ekman theory overestimates the reduction of growth rate at large drag due to overestimating Ekman pumping. This occurs because, as the friction coefficient $\gamma$ increases, the increased shear and boundary layer thickness, along with finite stratification, weaken the near-bottom velocity and vorticity $\zeta_{b}$ [Eq. (12); Fig. 11]. The use of interior vorticity to approximate the near-bottom value in the theory thus leads to large errors at large drag (Figs. 10a3,c3,d3). In other words, the baroclinic instability loses sensitivity to the friction factor (Fig. 8a) because the stratified Ekman layer tends to limit the increases in bottom stress and Ekman pumping.

In the Eady-Ekman theory, the strength of Ekman pumping is solely represented by the thickness $d$ in the friction parameter $\Delta_{E}[=d k / S$; Eq. (6)]. This dependence comes from $w=d \zeta_{v^{\prime} \max }$ [Eq. (4)], where $d\left(=\gamma / f_{0}\right)$ sets the proportionality between pumping velocity and interior vorticity. We have seen that $d \zeta_{v^{\prime} \text { max }}$ overestimates the actual vertical velocity at large drag, and this is explained by $\zeta_{v^{\prime} \max } \gg \zeta_{b}$ (Figs. 10c3,d3). However, if we insist on expressing $w$ in term of the interior vorticity as $w=D \zeta_{v^{\prime} \max }$, the problem may be attributed to an overestimation of the boundary layer thickness $D=d=$ $\gamma / f_{0}$, because, with the weakening of near-bottom velocity, the bottom stress should not scale linearly with $\gamma$. A more sensible representation of the thickness may be the stratified Ekman layer scaling $D=d_{\text {strat }}=u_{*} / \sqrt{N f_{0}}$ (Pollard et al. 1973; McWilliams et al. 2009), where the thickness explicitly depends on bottom stress and interior stratification $N$.

Based on the above argument, we make a correction to the Ekman pumping velocity $\left(w=d_{\text {strat }} \zeta_{v^{\prime} \max }\right)$ and introduce a rescaled friction parameter: 

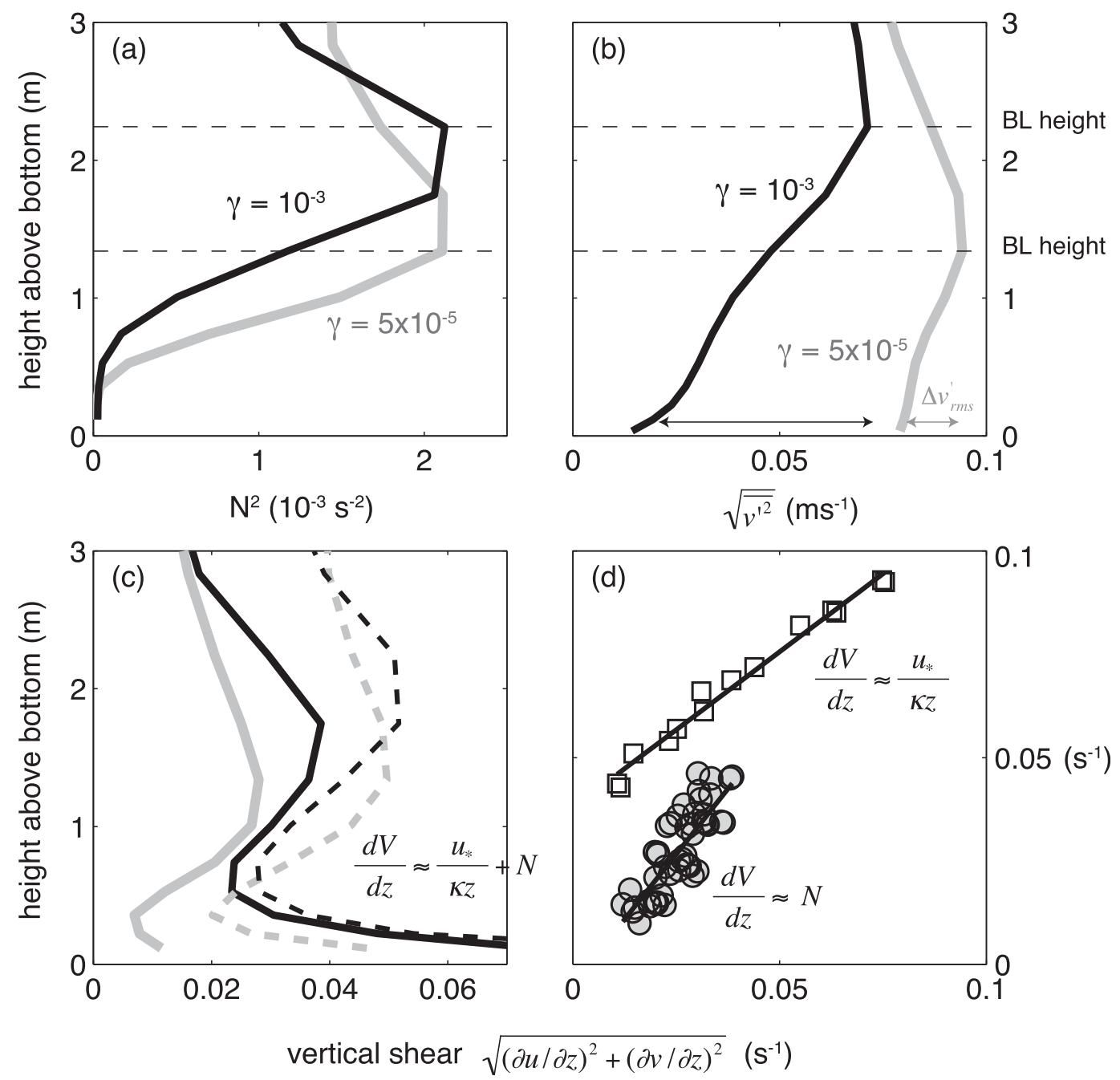

FIG. 11. Near-bottom ( $\leq 3 \mathrm{~m}$ ) structure of (a) $N^{2}$, (b) $v_{\text {rms }}^{\prime}$, (c) vertical shear, for two cases with contrasting friction factors $\left[\gamma=5 \times 10^{-5}\right.$ (gray) and $10^{-3}$ (black) $\mathrm{m} \mathrm{s}^{-1}$ ]. These quantities are rms values (i.e., in alongshore direction) and averaged over the mean flow center. In (a) and (b), the horizontal dashed lines indicate the boundary heights for the two cases. In (c), the scaling of shear for a stratified Ekman layer [Eq. (12)] is indicated by the dashed curves. (d) Comparison of the law of the wall scaling for the bottom grid point (squares) and stratified scaling (shear $\sim N$ ) for the upper half of the boundary layer (circles).

$$
\Delta_{E}^{\prime}=d_{\text {strat }} k / S=\left(u_{*} / \sqrt{N f_{0}}\right)(k / S)
$$

This correction weakens the Ekman pumping at large drag because $d_{\text {start }} \ll \gamma / f_{0}$. Using this corrected friction parameter, the model-theory discrepancy at large drag is reduced (e.g., Fig. 8a versus Fig. 8b). The overall agreement is also significantly improved, as the model results tend to collapse onto the theoretical curves (Figs. 8b,d,f), except for a few cases with largest drag. Quantitatively, the correction reduces the root-meansquare deviation for growth rate and bottom wave speed by a factor of 3 and 1.7 , while the deviation for wavelength remains comparable $(\sim 0.4$; around $8 \%$ of the mean wavelength). Although the correction is empirical, the improved agreement provides support for attributing the instability's loss of sensitivity to friction factor at large drag to the boundary layer response, which tends to limit the strength of bottom stress and Ekman pumping.

\section{Influences of bottom slope}

\section{a. Structure and growth rate}

Finally we investigate the effects of bottom slope (Group 3 in Table 1). As in Figs. 5 and 7, we compare the instability structure and time series of energy level, $K_{E}$ budget, and growth rate among three cases (Fig. 12). 

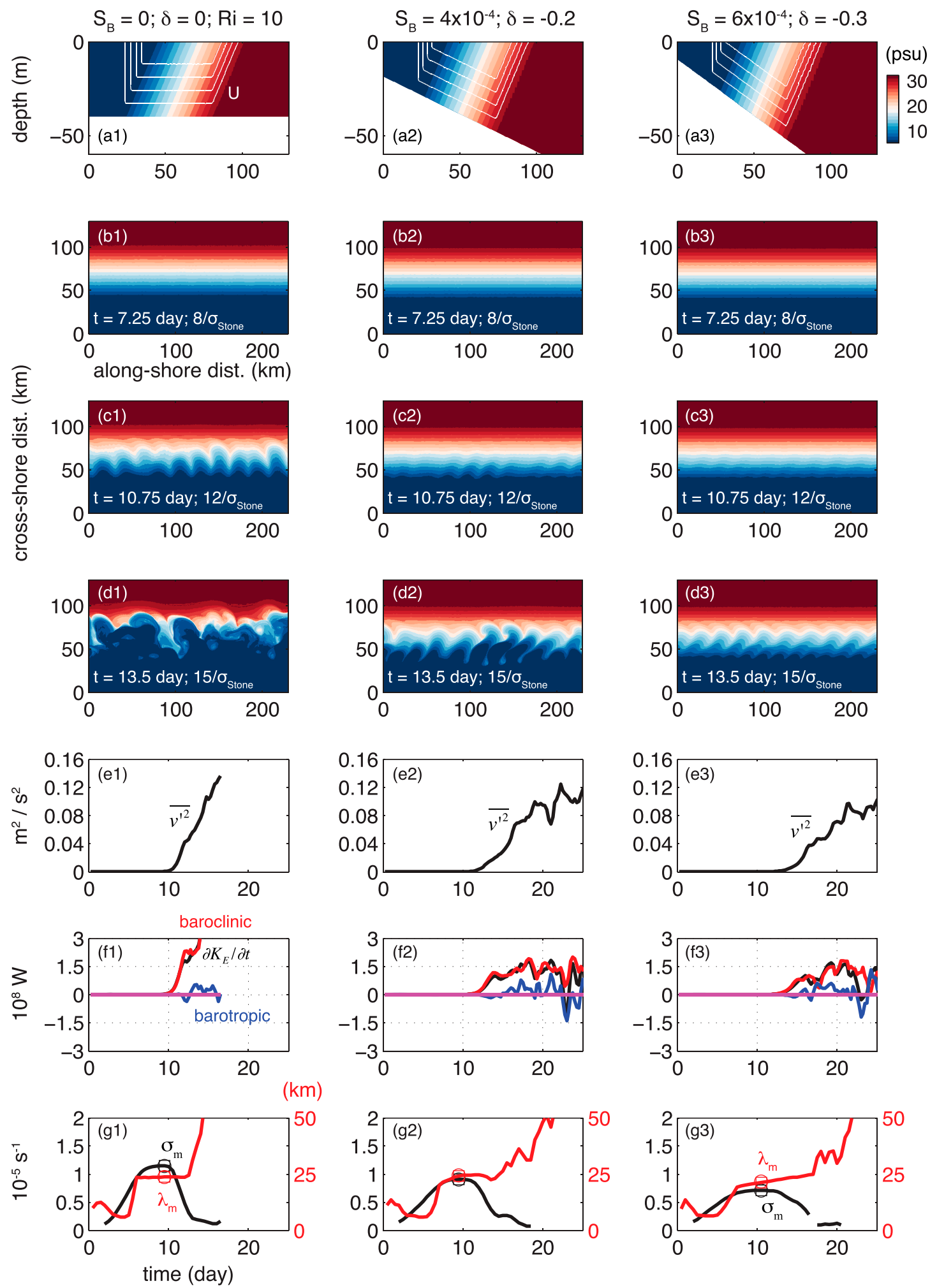

FIG. 12. As in Fig. 5, but here Ri is held fixed $(=10)$. The three cases differ only in the bottom slope $\left(S_{B}=0,4 \times 10^{-4}\right.$, and $6 \times 10^{-4} ; H=40 \mathrm{~m}$; cases 22,24 , and 26 in Table 1$)$, corresponding to $\delta=0,-0.2$, and -0.3 . 
These three cases (runs 22, 24, 27) differ only in the bottom slope (Fig. 12a). They have identical isopycnal slopes, with $\mathrm{Ri}=10$ (same $M^{2}$ and $N^{2}$ ). The basic flow has the same width of $40 \mathrm{~km}$, centered at the mean depth of $40 \mathrm{~m}$. As before, we quantify the instability growth rate and wavelength across half of the mean flow (i.e., centered at $H=40 \mathrm{~m}$ ). The simulations are carried out with viscosity set to zero.

Increasing bottom slope tends to suppress the growth of baroclinic instability (Fig. 12). The growth in $K_{E}$ is primarily driven by baroclinic energy conversion (Fig. 12f), suggesting the dominance of baroclinic instability as in the other cases studied. As the bottom slope increases, the amplitude of the disturbances in surface salinity decreases (e.g., Figs. 12c1-c3,d1-d3). This suppression of instability is clearly reflected in the time series where the levels of $\overline{v^{2}}$ and growth rate both decrease with increasing slope (Figs. 12e1e3,g1-g3). In particular, the growth rate of the most unstable mode $\sigma_{m}$ (black squares) decreases from $1.2 \times 10^{-5}$ to $0.62 \times 10^{-5}\left(\mathrm{~s}^{-1}\right)$ as the slope increases. The corresponding wavelength $\lambda_{m}$ also shortens from 23.8 to $18.6(\mathrm{~km})$.

\section{b. Testing Blumsack and Gierasch's theory}

We evaluate the utility of a quasigeostrophic theory, here the BG72 model for slope effects, by comparing the predictions against the model results (Fig. 13). Recall that the steepness of bottom slope is measured relative to isopycnal slope $\left(\delta=S_{B} / S\right)$. The term $\delta$ is negative because these two slopes are tilted in the opposite way.

The theory captures the decrease in growth rate, shift to shorter wavelength, and increase in bottom wave speed with increasing slope ratio $|\delta|$ (Fig. 13). Over relatively gentle bottom slopes (i.e., $|\delta| \leq 0.5$ ), the agreement is very good for the wave speed and wavelength. The theory tends to overestimate the growth rate by a mean error of around $30 \%$ (Fig. 13a). However, overall, there is a reasonable agreement between BG72 theory and the numerical model results for $|\delta| \leq 0.5$.

The decrease in growth rate with increasing slope may be understood by noting that an offshore displacement along the lower boundary would force a buoyant water parcel downward, unfavorable to the release of potential energy (BG72). The instability selects a shorter wave mode in order to counter the speedup of bottom waves (see section 2a). However, it should be noted that the predictability of the theory appears to deteriorate as $|\delta|$ increases. The error of growth rate estimates increases from $14 \%$ to over $75 \%$ as $\delta$ changes from 0 to -0.6 (Fig. 13a). The model-theory deviations in estimates of wave speed and wavelength also increase for large $|\delta|$ (Figs. 13b,c), suggesting missing dynamics in the theory
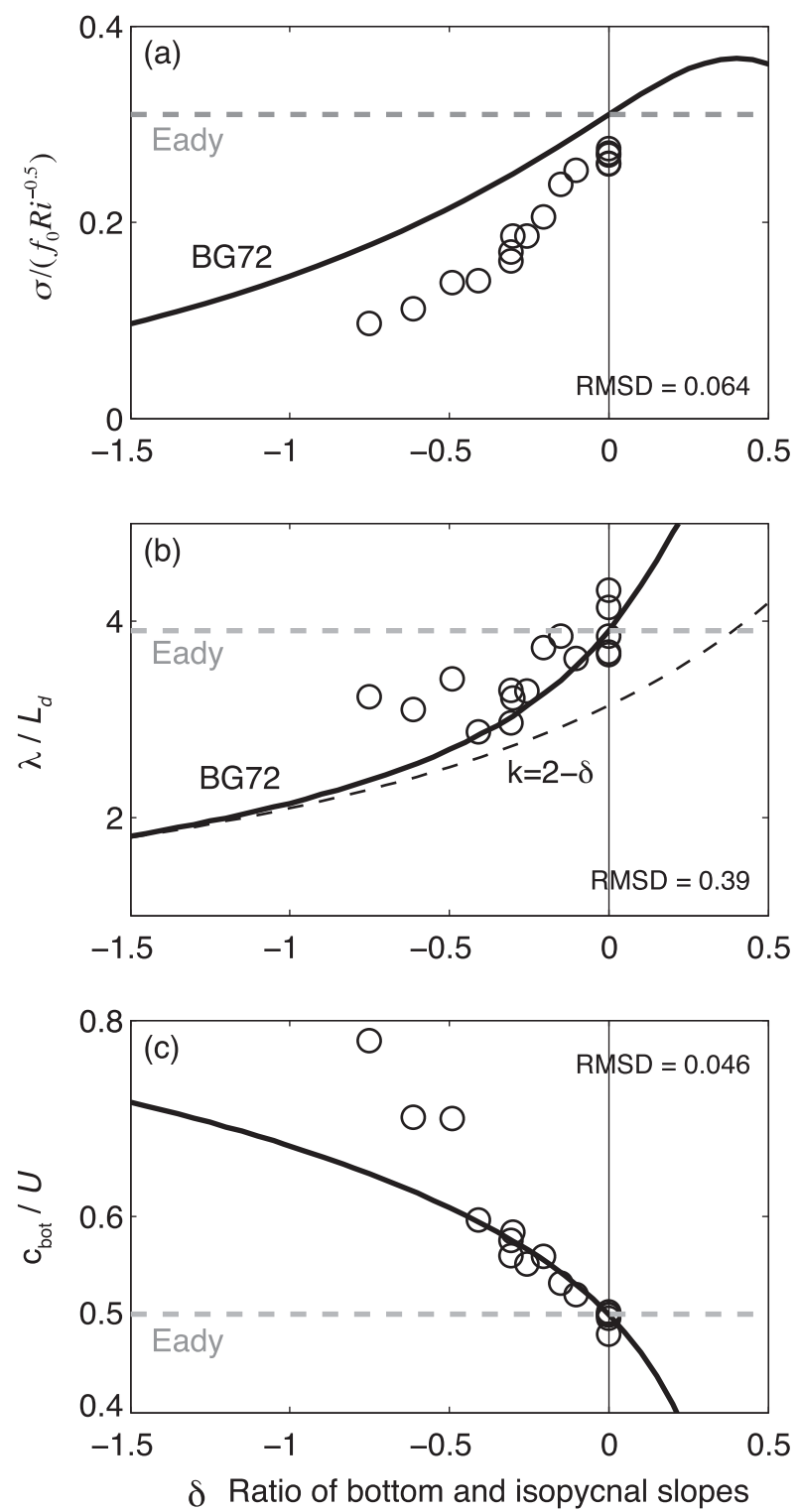

FIG. 13. Evaluation of BG72 theory for the slope effect on the baroclinic instability. (a) Growth rate, (b) wavelength, and (c) bottom wave speed are plotted against the slope ratio $\delta$ [Eq. (10)]. These quantities are dimensionless as in Fig. 8. The model-derived quantities are the open symbols. Black curves indicate BG72 theory. The horizontal gray dashed lines indicate the Eady solution. In (b), the black dashed curve $(k=2-\delta)$ is an approximate marginal stability condition obtained by matching top and bottom wave speed (see section 2a). The RMSDs for the model-theory comparisons are given.

over steep slopes, which is explored further in the next subsection.

\section{c. Influences of horizontal shear and eddy tilting}

We suggest that the decrease in predictive skill of the theory as the bottom slope steepens is linked to the 

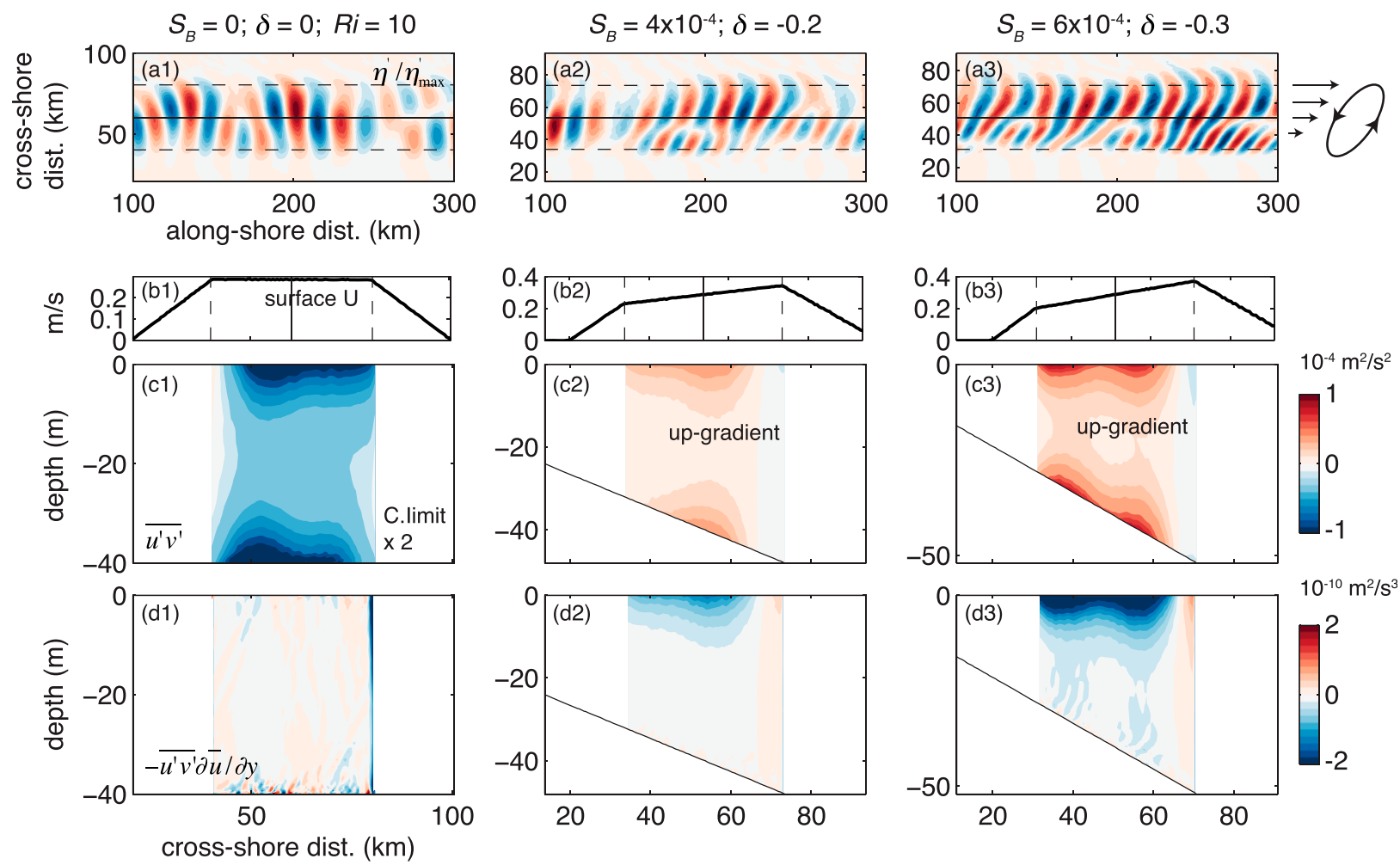

$10^{-10} \mathrm{~m}^{2} / \mathrm{s}^{3}$

FIG. 14. Comparisons of (a) eddy orientation, (b) cross-shore profile of initial surface mean flow, (c) eddy momentum flux $\overline{u^{\prime} v^{\prime}}$, and (d) barotropic energy conversion $\left(-\overline{u^{\prime} v^{\prime}} \partial \bar{u} / \partial y\right)$, for the three cases shown in Fig. 12; (a), (c), and (d) are taken at the maximal growth rate (squares in Fig. 12g). Shown in (a) is the top view of pressure perturbations normalized by the maximal value. In (d), only the dominant conversion [first term on the right in Eq. (B1)] is plotted. In (c1), the contour limits are multiplied by two for better visualization. At the top right corner, a schematic is shown to illustrate how horizontally sheared mean flow [arrow lines; (b2) and (b3)] tilts an eddy (a tilted ellipse) that leads to predominantly negative energy conversion in (d2) and (d3).

increase in influence of horizontal shear which was not included in the BG72 model. We first recognize that a balanced mean flow with zero bottom velocity will have increased horizontal shear as the bottom slope steepens, due simply to the depth variation $\left[\partial U / \partial y=\left(M^{2} / f_{0}\right) \partial H / \partial y=\right.$ $\left.-\left(M^{2} / f_{0}\right) S_{B}\right]$. However, in BG72, this effect is assumed negligible for mild slopes.

The influence of horizontal shear on the eddy field is illustrated in Fig. 14, comparing three cases that differ only in bottom slope. The panels from top to bottom are the top view of fluctuating surface elevation $\eta^{\prime}$, surface mean flow speed as a function of cross-shore distance, and cross-shore profiles of eddy momentum fluxes $\overline{u^{\prime} v^{\prime}}$ and barotropic energy conversion in the eddy kinetic energy budget [Eq. (B1)]. Parameter $\eta^{\prime}$ is a proxy for geostrophic eddy orientation (i.e., pressure field). Figure $14 \mathrm{~d}$ shows only the dominant term of the conversion that is associated with horizontal shear $\left(-\overline{u^{\prime} v^{\prime}} \partial \bar{u} / \partial y\right)$.

Horizontal shear at the surface clearly increases with bottom slope (Fig. 14b). As the slope steepens, the surface eddies are increasingly tilted in northeastsouthwest direction (toward offshore and $+x$ ) by the mean horizontal shear (Fig. 14a). This eddy orientation leads to predominantly positive eddy momentum fluxes across the mean flow (Figs. 14c2,c3). The magnitude of $\overline{u^{\prime} v^{\prime}}$ increases with the tilt and hence with the bottom slope. The positive $\overline{u^{\prime} v^{\prime}}$ due to tilted eddies occurs mostly over the region with $\partial U / \partial y>0$. This means that the momentum fluxes are largely upgradient, acting to convert the eddy kinetic energy back to the mean flow (i.e., negative energy conversion in Figs. 14d2,d3) and therefore reducing the instability growth.

The loss of eddy kinetic energy via upgradient momentum fluxes due to tilted eddies is further supported by diagnosis of the energy equation. In Fig. 15, we plot the volume-integrated barotropic energy conversion against the slope ratio $\delta$ for all cases. To compare among different basic states, we normalize the barotropic conversion by the dominant (positive) eddy kinetic energy source of baroclinic conversion. The conversion ratio is evaluated at the time of maximal growth rate, but the result is robust within a window of a few days before finite-amplitude effects become significant. Barotropic energy conversion during maximal growth is negative 
for all cases with a sloping bottom (Fig. 15). Over relatively gentle slopes $(|\delta| \leq 0.5)$, the conversion ratio scales positively with $\delta$ (as indicated by the linear fit), meaning that the barotropic conversion becomes more negative as the bottom slope steepens. This pattern is consistent with the interpretation in Fig. 14, that increasing eddy tilting by bottom-slope-induced horizontal shear converts more eddy kinetic energy back to the mean. In other words, the bottom-slope-induced horizontal shear acts as an energy sink to suppress the instability growth. Because BG72 theory did not include this effect, the theory increasingly overestimates the growth rate as the bottom slope steepens (Fig. 13a). The positive relationship between conversion ratio and $\delta$ appears to break down at steep slopes, when $|\delta|$ exceeds around 0.5 (Fig. 15). Detailed analyses of routes of energy transfer and the potential influences of barotropic instability are beyond the scope of this work and thus left for future studies.

\section{Summary and discussion}

The main objective of this study is to examine the utility of the Eady-type theories in understanding baroclinic instability in coastal buoyancy-driven flows. We focus on the effects of nongeostrophy, boundary dissipation, and bottom slope.

Not surprisingly, with an Eady-like basic state and in the absence of viscosity (e.g., valid for interior flow), bottom slope, and horizontal shear, the coastal baroclinic instability shows growth rate and wave properties consistent with the Eady theory. The governing parameter is the Richardson number Ri [ $=N^{2} f_{0}^{2} M^{-4}=\mathrm{Ro}^{-2}$; Eq. (2)]. When the basic state is in the quasigeostrophic regime $(\mathrm{Ri}>5)$, the ROMS-derived growth rate $\sigma_{m}$ and wavelength $\lambda_{m}$ for the most unstable mode agree with Eady predictions of $0.31 f_{0} \mathrm{Ri}^{-1 / 2}$ and $3.9 L_{d}$ (Fig. 6). QG or not, the growth rate varies inversely with Ri because a steeper isopycnal associated with a smaller $\mathrm{Ri}$ (e.g., decreasing $N^{2}$ while holding $M^{2}$ and $f_{0}$ constant) allows a horizontally displaced parcel to rise higher and thus releases more potential energy [Eqs. (1) and (3)]. This study also confirms that the growing instability in the numerical experiments can be interpreted as mutual reinforcement of boundary-trapped Rossby waves (e.g., Hoskins et al. 1985). Specifically, we find evidence for wave phase locking, with counterpropagating waves that translate at the same apparent speed of $U / 2$, and westward phase tilt, with top-bottom phase difference near $\pi / 2$, allowing waves to be mutually amplified (Fig. B1).

As Ri decreases, the steepened isopycnal slope gives rise to nonnegligible vertical motion and hence increases the non-QG effects. Stone's (1966) theory is shown to

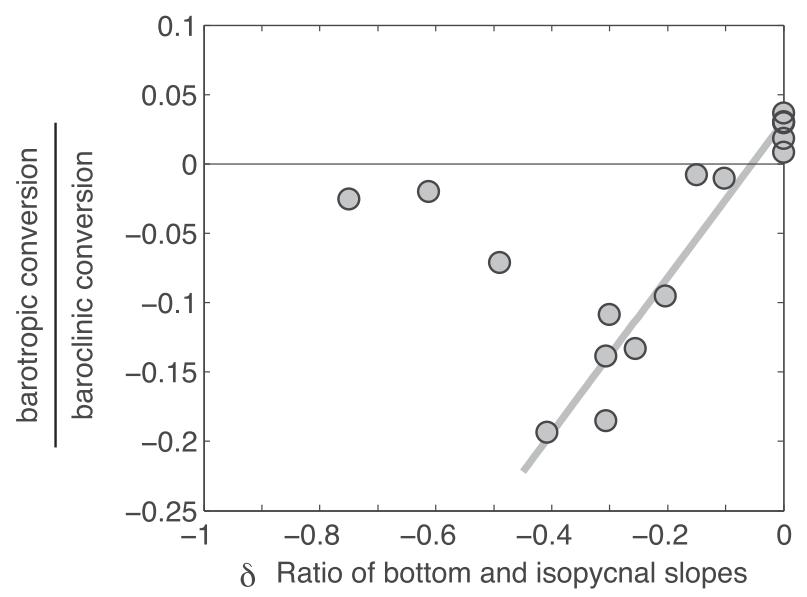

FIG. 15. Volume-integrated barotropic energy conversion against the slope ratio $\delta$ for all cases with a bottom slope (cases 22 to 34). The barotropic conversion is normalized by baroclinic conversion $\left[-C\left(K_{E}, K_{M}\right) / C\left(P_{E}, K_{E}\right)\right.$; Eq. (B2)] and is computed at maximal growth rate. Note that the barotropic conversion is negative for all cases with a nonzero bottom slope. The gray line is a linear fit for $\delta>-0.5\left(R^{2}=0.93\right)$, indicating a positive relation between the bottom slope and barotropic energy conversion (see section 5c).

give reasonably accurate nongeostrophic corrections for $\mathrm{Ri}>1$ : compared with the Eady mode, the nongeostrophic baroclinic instability has a lower growth rate and longer wavelength as predicted by Eq. (3) (Fig. 6). The shift to a longer wave mode is consistent with Nakamura's (1988) explanation. That is, nongeostrophy makes the boundarytrapped waves shallower (Fig. B1e). The instability thus tends to select longer waves to maintain the vertical coupling.

Contrary to the agreement with Stone's nongeostrophic theory, the model results suggest that, even under idealized settings, the Eady-Ekman and BG72 theories have inherent limitations, due to the neglect of boundary layer responses and horizontal shear, respectively.

For boundary dissipation, the analyses support the conceptual model put forth by the Eady-Ekman theory, but there are problems at large drag. Dissipation concentrates in a thin boundary layer which affects the instability via inducing Ekman pumping at the lower boundary of the interior flow (Fig. 10). The theory introduces a bottom friction parameter $\Delta_{E}$ [Eq. (6)], which assumes that the strength of pumping $w$ increases linearly with the friction factor $\gamma$ [Eq. (4)]. Qualitatively, the Eady-Ekman theory captures the general tendency of decreasing growth rate and increasing wavelength when bottom friction $\Delta_{E}$ increases (Figs. 8a,c). However, the theory overestimates the growth rate reduction at large $\Delta_{E}$ (e.g., by $70 \%$ at $\Delta_{E}=2.8$ ). The problem lies in that the bottom stress does not increase linearly with $\gamma$. 
Instead, the increased vertical shear and boundary layer thickness, along with the presence of stratification, weakens the near-bottom velocity [Fig. 11 and Eq. (11)]. This boundary layer response buffers the increase of bottom stress, which in turn limits Ekman pumping and the slowdown of bottom waves [Eq. (7)]. An empirical correction of Ekman pumping in $\Delta_{E}$ [Eq. (13)] significantly improves the model-theory comparison (Figs. 8b,d,f), highlighting the key role of Ekman pumping in modifying the instability properties.

For bottom slope, BG72 theory provides reasonable predictability over gentle slopes. However, agreement between the simulations and theory decreases as the bottom slope increases, and the slope-induced horizontal shear in the basic state, which is not accounted for in the theory, becomes significant. A key parameter here is the slope ratio $\delta$ [Eq. (10)], for which we consider only negative values with the bottom and isopycnals tilted in opposite directions. Over a relatively gentle bottom slope $(|\delta|<0.5)$, as $|\delta|$ increases, the observed decrease in wavelength and increase in bottom wave speed are well represented by BG72 (Figs. 13b,c). The theory also captures the reduction in growth rate.

Bottom slope induces horizontal shear in the basic state via the thermal wind relation. This shear increases with bottom slope. As $|\delta|$ increases, the sheared flow tilts the eddies and increasingly favors converting $K_{E}$ back to the mean flow (Fig. 14). The slope-induced horizontal shear therefore acts as an energy sink to suppress the instability, leading to the increased theory-model discrepancies.

\section{a. Interpreting the friction and slope parameters and scale selection}

We now expand on the implications and limitations of this work. The analyses in this study clearly show the dependence of instability properties on the friction parameter $\Delta_{E}$ and slope ratio $\delta$. In addition to their links to bottom buoyancy perturbations (section $2 \mathrm{a}$ ), the physical meaning of these two parameters may be further illuminated from a geometric (or potential vorticity) perspective. Using Eqs. (4), (7), and (11), we find, for flat bottom with friction,

$$
\left.\left[\left(w / v^{\prime}\right) / S\right]\right|_{z=0}=i \Delta_{E}=i(d k / S),
$$

and, for sloping bottom with zero friction,

$$
\left.\left[\left(w / v^{\prime}\right) / S\right]\right|_{z=0}=\delta=S_{B} / S .
$$

These expressions make explicit the fact that, in terms of scales, $\Delta_{E}$ and $\delta$ both represent the slope of the bottom parcel trajectory $\left(w / v^{\prime}\right)$, relative to the isopycnal slope $S$.
The importance of parcel trajectory can be understood in the context of vortex stretching/squashing. Recall from Fig. 1a that the generation of boundary-trapped waves in the Eady model can be interpreted via stretching/squashing of a vortex element. An offshore displacement along the lower boundary tends to induce stretching and cyclonic circulation, leading to wave propagation in the $+x$ direction (Fig. 1c).

We suggest, using geometric arguments, that when the friction effect is included, Ekman pumping reduces vortex stretching due to the upward-seaward bottom parcel movement ( $w>0, v^{\prime}>0$; thick arrow in Fig. 2a). Conversely, the presence of a bottom slope enhances the stretching by requiring the parcel to move parallel to the topography $\left(w<0, v^{\prime}>0\right.$ in Fig. $\left.2 b\right)$. Therefore, in the case of friction (bottom slope with $\delta \leq 0$ ), the parcel movements effectively reduce (enhance) the background PV gradients and thus decrease (increase) the bottom wave speed (Figs. 3b,d). The slowdown (speedup) of the bottom wave then forces the instability to select a longer (shorter) wave mode to maintain the phase lock (Figs. 3a,c).

While $\Delta_{E}$ and $\delta$ can both be interpreted as slope ratios, we cannot make a quantitative analogy between the friction and bottom slope effects. Based on the above PV argument, bottom Rossby waves will not exist when the slope ratio becomes one, because a horizontally displaced vortex element cannot be stretched/squashed. Without the bottom wave to interact and resonate with the top wave, an instability will not grow. Indeed, we find that the instability growth rate vanishes with $\delta \geq 1$ as shown in Fig. 3c.

However, such a behavior cannot be seen for the frictional effects. In Fig. 3a, there is still finite growth rate with $\Delta_{E} \geq 1$. The main difference may lie in that, in the case of bottom friction, $w$ and $v^{\prime}$ are $90^{\circ}$ out of phase [Eq. (14a)]. This relation simply reflects that the maximum pumping occurs where the spatial gradient of $v^{\prime}$ and thus divergence of Ekman transport is maximal. Therefore, the maximal $w$ and $v^{\prime}$ are staggered in both space and time, contrary to the collocated $w$ and $v^{\prime}$ extrema over a sloping bottom.

\section{b. Linkage with prior studies}

The findings of this work have clear linkages with prior studies. For example, Brink and Cherian (2013) and Hetland (2017) both invoked frictional spindown as key processes in their modeling studies on the baroclinically unstable tidal mixing fronts and coastal currents. Brink and Cherian (2013) proposed a scaling for frictionally damped eddy kinetic energy, based on a mismatch of spindown and eddy turnover time scales. Hetland (2017) used similar time-scale mismatch arguments to isolate the 
cases where frictional processes are negligible. The presence of spindown is clearly supported by this study. Not surprisingly, bottom Ekman transport is a robust feature in all frictional cases (Fig. 10), and the nearly inviscid interior is forced by Ekman pumping. However, this study points out further that the frictional influence on the instability does not increase proportionally with the friction factors (linear or quadratic; Figs. 8a,c,e). The increased vertical shear across the boundary layer tends to weaken the near-bottom flow, thereby limiting the increase in bottom stress and Ekman pumping. This buffering effect of the boundary layer may explain why the eddy length scales became insensitive to the linear friction factor as reported by Brink and Cherian (2013, their Fig. 7). Moreover, we expect the weakening in sensitivity to the friction factor to be a generic process in coastal flows. Thus, parameterizations for eddy fluxes that are based on Eady-type linear theories [e.g., following Stone's (1972) approach] will likely need different friction-factor dependences at least for small and large drag regimes.

For bottom slope effects, several recent studies have incorporated bottom slope dependence into the scalings for eddy length scales, growth rate, and buoyancy fluxes (e.g., Brink 2012; Zhang and Gawarkiewicz 2015; Spall 2004). The general form for some of the scalings may be deduced directly from the BG72 theory. For example, Brink (2012) scaled the eddy length and growth rate of baroclinically unstable tidal mixing fronts as $\lambda / L_{d} \sim$ $1 /\left(1+b_{2} s\right)$ and $\sigma /\left(f_{0} \mathrm{Ri}^{-0.5}\right) \sim 1 /\left(1+b_{1} s\right)$, respectively, where $s=-S_{B} N / f_{0}$ is the slope Burger number and $b_{1}, b_{2}$ are empirical constants. Zhang and Gawarkiewicz (2015) applied similar scalings to characterize unstable shelfbreak fronts. We can see immediately from the above functional form that bottom slope $S_{B}$ exerts a stabilizing effect and makes the most unstable waves shorter $(s>0)$, consistent with BG72 (Fig. 13). If we use BG72's approximate marginal stability condition (i.e., matching top and bottom wave speed; section $2 \mathrm{a})$ of $\tilde{k}=(2 \pi / \lambda) L_{d}=2-\delta$ and note that $\delta=S_{B} N^{2} / M^{2}=-s \sqrt{\mathrm{Ri}}$, we obtain $\lambda / L_{d}=$ $\pi /\left[1+\left(\mathrm{Ri}^{1 / 2} / 2\right) s\right]$, yielding a similar functional form as above. The consistency thus provides a theoretical basis for the prior scalings.

Note however that our study also emphasizes the limitations of BG72. As the bottom slope steepens, the slope-induced horizontal shear leads to increasing and nonnegligible differences between ROMS-derived and BG72 growth rates. This result therefore suggests caution in applying the existing scalings to systems with steep topography. Taking $|\delta|=0.5$ as a limit (Fig. 13) and $\mathrm{Ri}=5$ for nearly $\mathrm{QG}$ basic flows, we may expect BG72 theory to be applicable for $s \leq 0.2$, beyond which the slope-induced horizontal shear would lead to significant errors (Fig. 13a).
The tight coupling between the bottom slope and horizontal shear shown in this study also has implications for coastal current stability. In a recent attempt to understand why unstable coastal currents were rarely reported in the literature, Hetland (2017) proposed that, due to the proximity to a coastal boundary, the width of the coastal currents may be too narrow for baroclinic eddies to develop. This conclusion was drawn primarily from ROMS simulations showing instabilities being inhibited over a range of parameters, which contradicts the finite growth rate predicted by BG72 (e.g., finite $\sigma$ for $|\delta| \leq 2$ in Fig. 3c). Considering that, even without invoking effects such as bottom drag, nongeostrophy, and width, the slope-induced horizontal shear alone can reduce $\mathrm{BG} 72$-predicted growth rate by more than $50 \%$ at $|\delta| \sim 0.5$ (Fig. 13a), it seems plausible that some of the inhibited growth in Hetland (2017) may be attributed to horizontal shear. However, the width dependence and horizontal shear are hard to separate as they are both scaled by the coastal current width. Nevertheless, the coupling of slope and horizontal shear is another stabilizing factor that requires further consideration.

It is worth noting that horizontal shear stabilization is well established in the atmospheric science literature (referred to as the "barotropic governor"). James (1987) suggested that the eddy energy transfer from potential to mean kinetic energy that strengthens the atmospheric eddy-driven jets can be hindered when the horizontal shear of a barotropic jet becomes large enough to reduce the meridional coherence of the eddy field. There appears to be differences between the barotropic governor and slope-induced horizontal shear. The former is part of a nonlinear feedback loop (e.g., Nakamura 1993), whereas the latter can operate at the linear stage of instability growth (Fig. 13). Yet, their mechanisms are the same: horizontal shear affects the eddy orientation and thus the eddy energy conversion rate [Eq. (B2)]. Therefore, insights gained from the barotropic governor effect could benefit further investigations on the slopeshear coupling.

Finally, this study has focused on individual effects, aiming to solidify our fundamental understanding of baroclinic instability over continental shelves. The combined effects of bottom drag, bottom slope, nongeostrophy, and horizontal shear are left largely unaddressed (except for some limited analyses on slope-horizontal shear coupling). When these effects are combined to represent more realistic basic flows (Fig. 4h), it is clearly important to identify the parameter regimes over which one particular effect may dominate. Also, combining the effects could potentially introduce additional processes that merit further investigation. For example, considering bottom drag over a sloping bottom allows bottom 
stress to adjust via buoyancy shutdown (e.g., MacCready and Rhines 1991; Brink and Lentz 2010). This may further limit Ekman pumping and thereby modify the interior instability. The mixed barotropic-baroclinic and symmetric instabilities could come into play when horizontal shear and nongeostrophy are included (e.g., Allen and Newberger 1998; Wenegrat et al. 2018). Additionally, forcing by wind and tides is expected to modify the coastal baroclinic instability through mixing and supplying/removing available potential energy. In view of the scope of the full problem, this study represents a small step toward a comprehensive understanding of instabilities over continental shelves.

Acknowledgments. This work is supported by the Ministry of Science and Technology of Taiwan through grants MOST 106-2628-M-002-006-MY3 and MOST 108-2611-M-002-022-MY4. Part of the computing resources was provided by the Center for Advanced Study in Theoretical Sciences at National Taiwan University. Lerczak was supported by U. S. National Science Foundation Grants OCE-1260394 and OCE-1829979. We acknowledge the helpful comments provided by Yan Wang (HKUST) and an anonymous reviewer.

\section{APPENDIX A}

\section{Eady-Type Models}

\section{a. Eady model (inviscid problem without topography)}

The Eady problem is briefly reviewed here. We closely follow the notation of Vallis (2017). Skipping the linearization of the quasigeostrophic potential vorticity conservation equation, we go straight to an equation set applicable to Eady's simple basic flow. For detailed derivations, refer to Vallis [2017, chapter 9, Eqs. (9.62)-(9.78)].

The linearized QGPV equation below describes pure alongshore (zonal) advection of QGPV perturbations

$$
\left(\frac{\partial}{\partial t}+\Lambda z \frac{\partial}{\partial x}\right)\left(\nabla^{2} \psi^{\prime}+\frac{f_{0}^{2}}{N^{2}} \frac{\partial^{2} \psi^{\prime}}{\partial z^{2}}\right)=0
$$

where the term in the first parentheses is an advection operator [mean flow $\bar{u}(z)=\Lambda z ; z=0$ at the bottom], and the second is the QGPV perturbation $q^{\prime}$ due to relative vorticity and vortex stretching, with $\psi^{\prime}$ being the perturbation streamfunction, $\left(u^{\prime}, v^{\prime}\right)=\left(-\partial \psi^{\prime} / \partial y, \partial \psi^{\prime} / \partial x\right)$. An overbar refers to an alongshore mean and a prime denotes a perturbation. Equation (A1) incorporates the simplifications associated with the Eady basic flow. The purely alongshore mean flow $\bar{u}$ is balanced and horizontally uniform, has constant vertical shear, constant stratification, constant depth, and is on an $f$ plane (Fig. 1a). Hence, the following parameters are all constant: buoyancy frequency $N\left(N^{2}=\partial \bar{b} / \partial z\right.$; where $b$ is buoyancy), cross-shore buoyancy gradient $\left(M^{2}=-\partial \bar{b} / \partial y\right)$, Coriolis parameter $f_{0}$, thermal wind shear $\left(\Lambda=M^{2} / f\right)$, and the isopycnal slope $\left(S=M^{2} / N^{2}\right)$. Note also that the crossshore advection of the background PV gradient $\left(v^{\prime} \partial \bar{q} / \partial y\right)$ does not appear in Eq. (A1). This is followed from $\partial \bar{q} / \partial y=-\bar{u}_{y y}-\left(f_{0}^{2} / N^{2}\right) \bar{u}_{z z}=0$ (subscripts denote partial derivatives) as the linearly sheared mean flow has zero curvature.

In a reentry channel, plane-wave solutions of the form $\psi^{\prime}=\operatorname{Re} \Phi(z) \sin l y e^{i k(x-c t)}$ for Eq. (A1) can be found [imposing $\psi^{\prime}(y=0, L)=0$ ]. The waves have an exponential structure in the vertical. The general solution is $\Phi(z)=A \cosh (\mu \tilde{z})+B \sinh (\mu \tilde{z})$, where $A$ and $B$ are undetermined coefficients, $\tilde{z}(=z / H)$ is a scaled height, and $\mu\left(=\sqrt{k^{2}+l^{2}} L_{d}\right)$ is the horizontal wavenumber normalized by the deformation radius $L_{d}\left(=N H / f_{0}\right)$. The top and bottom boundary conditions are $w(z=H)=$ $w(z=0)=0$. In Eady-type models, the boundary conditions come in through linearized buoyancy conservation

$$
\left(\frac{\partial}{\partial t}+\bar{u} \frac{\partial}{\partial x}\right) b^{\prime}+\frac{\partial \bar{b}}{\partial y} v^{\prime}+N^{2} w=0
$$

where the buoyancy perturbation $b^{\prime}$ can be expressed as $f_{0} \partial \psi^{\prime} / \partial z$ via the hydrostatic relation. The boundary conditions are

$$
\begin{aligned}
& w(z=H)=0:\left(\frac{\partial}{\partial t}+U \frac{\partial}{\partial x}\right) f_{0} \frac{\partial \psi^{\prime}}{\partial z}-\Lambda f_{0} \frac{\partial \psi^{\prime}}{\partial x}=0, \\
& w(z=0)=0:\left(\frac{\partial}{\partial t}\right) f_{0} \frac{\partial \psi^{\prime}}{\partial z}-\Lambda f_{0} \frac{\partial \psi^{\prime}}{\partial x}=0 .
\end{aligned}
$$

with the velocity scale $U=\Lambda H$. Plugging $\psi^{\prime}=$ $\operatorname{Re}\left[(A \cosh \mu \tilde{z}+B \sinh \mu \tilde{z}) \sin l y e^{i k(x-c t)}\right]$ into Eq. (A3) and nondimensionalizing velocity by $U$, spatial scale by $L_{d}$, and time scale by $L_{d} / U$, we find that the dimensionless phase speed $\tilde{c}(=c / U)$ for nontrivial solutions (i.e., $A, B \neq 0$ ) is given by

$$
\tilde{c}=\frac{1}{2} \pm \frac{1}{2}\left[1+\frac{4}{\mu^{2}}-\frac{4}{\mu} \operatorname{coth}(\mu)\right]^{1 / 2} \text {. }
$$

The phase speed $\left(\tilde{c}=\tilde{c}_{r} \pm i \tilde{c}_{i}\right)$ is complex. A wave mode with alongshore wavenumber $\tilde{k}\left(=k L_{d}\right)$ will grow exponentially with time at a rate of $\tilde{k} \tilde{c}_{i}$ if the imaginary part of the phase speed $\tilde{c}_{i}$ is nonzero. Because our focus is on the fastest growing baroclinic instability, throughout this work we assume perturbation variables are uniform in crossshore direction $(l \rightarrow 0 ; \mu=\tilde{k})$. The growth rate is then 

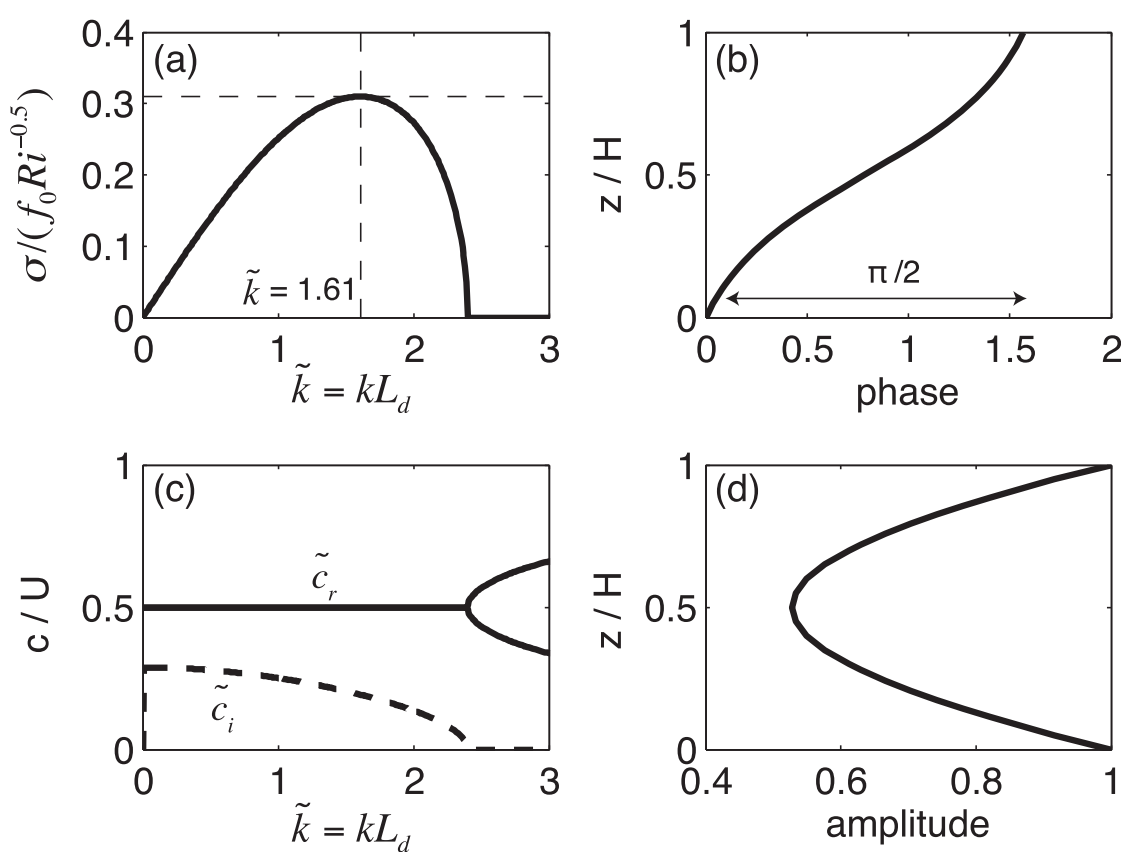

FIG. A1. The Eady solution properties (for $l=0$ ). (a) Growth rate and (c) phase speed are plotted against the dimensionless alongshore wavenumber $\tilde{k}$, according to Eqs. (A4) and (A5). In (a), the growth rate $\left(\sigma=k c_{i}\right)$ is made dimensionless by $f_{0} \mathrm{Ri}^{-0.5}$. The most unstable (Eady) mode, with $\sigma=0.31 f_{0} \mathrm{Ri}^{-0.5}$ and $\tilde{k}=k L_{d}=1.61$ [Eq. (1)], is indicated by the dashed lines. In (c), the solid and dashed curves are the real and imaginary part of the wave speed. (b), (d) The vertical structure of wave phase and amplitude for the most unstable mode. For the most unstable mode, the wave speed of $U / 2$ in (c), top-bottom phase difference of $\pi / 2$ in (b), and boundary-intensified wave amplitude in (d) are consistent with Hoskins et al.'s (1985) Rossby wave resonance interpretation (see section 2a).

$$
\tilde{\sigma}=\tilde{k} \tilde{c}_{i}=\left[\tilde{k} \operatorname{coth}(\tilde{k})-1-\tilde{k}^{2} / 4\right]^{1 / 2} .
$$

Using Eq. (A3b), we rewrite $\Phi=[\cosh (\tilde{k} \tilde{z})-$ $\left.(\tilde{c} \tilde{k})^{-1} \sinh (\tilde{k} \tilde{z})\right] A$. The vertical structure of wave amplitude and phase can be readily determined by $\left(\Phi \Phi^{*}\right)^{1 / 2}$ and $\tan ^{-1}[\operatorname{Im}(\Phi) / \operatorname{Re}(\Phi)]$, respectively (see below).

The properties of the Eady solution are shown in Fig. A1 (for $l=0$ ). The most unstable mode has a maximum $\tilde{\sigma}$ of 0.31 at $\tilde{k} \approx 1.61$ (Fig. A1a). Dimensionally, this corresponds to the Eady growth rate $\sigma=k c_{i}=$ $0.31 f_{0} \mathrm{Ri}^{-0.5}$ and wavelength $\lambda=(2 \pi / 1.61) L_{d}=3.9 L_{d}$ [as in Eq. (1); $\sigma$ is scaled by $\left.U / L_{d}=f_{0} \mathrm{Ri}^{-0.5}\right]$. In Fig. A1a, there is a well-known short-wave cutoff at $\tilde{k}=2.4$, beyond which the boundary-trapped waves cannot maintain phase locking (as the intrinsic phase speed is too slow) and their vertical coupling diminishes as wavenumber increases (i.e., $\Phi$ decays exponentially as $\tilde{k} \tilde{z}$ ). Over the unstable range $(\tilde{k}<2.4)$, the waves propagate in the $+x$ direction at half of the mean flow speed $(c / U=$ 0.5 in Fig. A1c), satisfying the phase-locking condition. The most unstable mode has the bottom wave leading the top by $\pi / 2$ (Fig. A1b) and is boundary-intensified (Fig. A1d). These features are consistent with Hoskins et al's (1985) interpretation of baroclinic instability in terms of Rossby wave interactions. This wave interpretation is described in section 2a of the main text.

\section{b. Boundary dissipation effect (Eady-Ekman model)}

As described in section $2 \mathrm{a}(2)$, in the Eady-Ekman model the boundary dissipative effect is represented by Ekman pumping that forces the inviscid interior at its lower boundary (i.e., top of the Ekman layer; Fig. 2a). The lower boundary condition of the original Eady problem [Eq. (A3b)] is replaced by Eq. (5). Following the same solution procedure, the phase speed becomes (again for $l=0$ )

$$
\begin{aligned}
\tilde{c}= & \frac{1}{2}\left[1-i \Delta_{E} \frac{\operatorname{coth}(\tilde{k})}{\tilde{k}}\right] \pm \frac{1}{2}\left\{\left[1-i \Delta_{E} \frac{\operatorname{coth}(\tilde{k})}{\tilde{k}}\right]^{2}\right. \\
& \left.-4\left(1-i \Delta_{E}\right)\left[\frac{\operatorname{coth}(\tilde{k})}{\tilde{k}}-\frac{1}{\tilde{k}^{2}}\right]\right\}^{1 / 2},
\end{aligned}
$$

where the friction parameter $\Delta_{E}$ [Eq. (6)] is a dimensionless measure of Ekman pumping strength. 
The vertical structure function is $\Phi=[\cosh (\tilde{k} \tilde{z})-(1-$ $\left.\left.i \Delta_{E}\right)(\tilde{c} \tilde{k})^{-1} \sinh (\tilde{k} \tilde{z})\right] A$. This solution gives the inviscid Eady solution when $\Delta_{E}=0$. We can calculate the growth rate $\tilde{k} \tilde{c}_{i}$ (as plotted in Fig. 3a) and vertical profiles of amplitude and phase with $\Phi(\tilde{z})$ as described above.

\section{c. Bottom slope effect (BG72)}

As with boundary dissipation, the bottom slope effect is incorporated into the Eady model through the lower boundary condition [see section $2 \mathrm{a}(3)$ ]. Equation (A3b) is now replaced by Eq. (9). The phase speed is

$$
\begin{aligned}
\tilde{c}= & \frac{1}{2}\left[1-\delta \frac{\operatorname{coth}(\tilde{k})}{\tilde{k}}\right] \pm \frac{1}{2}\left\{\left[1-\delta \frac{\operatorname{coth}(\tilde{k})}{\tilde{k}}\right]^{2}\right. \\
& \left.-4(1-\delta)\left[\frac{\operatorname{coth}(\tilde{k})}{\tilde{k}}-\frac{1}{\tilde{k}^{2}}\right]\right\}^{1 / 2},
\end{aligned}
$$

where $\delta$ [Eq. (10)] measures the steepness of a bottom slope relative to the isopycnal slope, and the vertical structure is given by $\Phi=[\cosh (\tilde{k} \tilde{z})-(1-$ $\left.\delta)(\tilde{c} \tilde{k})^{-1} \sinh (\tilde{k} \tilde{z})\right] A$. The growth rate is

$\tilde{\sigma}=\tilde{k} \tilde{c}_{i}=\left\{(1-\delta)[\tilde{k} \operatorname{coth}(\tilde{k})-1]-[\tilde{k}-\delta \operatorname{coth}(\tilde{k})]^{2} / 4\right\}^{1 / 2}$,

identical to the solution of BG72 [their Eq. (3.11)]. The growth rate in $\tilde{k}-\delta$ space is plotted in Fig. $3 c$. It is worth noting the similarity between the Eady-Ekman and BG72 models. Their lower boundary conditions and governing parameters have similar forms [Eqs. (5)-(7) versus Eqs. (9)-(11)] such that the solutions of phase speed [Eqs. (A6) and (A7)] are nearly identical (i.e., replacing $i \Delta_{E}$ by $\delta$ ). This analogy will be elaborated on further in section $6 a$.

\section{APPENDIX B}

\section{Metrics for Analyses}

We evaluate the properties of Eady-type theories against the numerical model results using the following metrics: the most unstable growth rate, wavelength, vertical structure of wave speed, phase, and amplitude. In addition, the energetics is examined via the volume-integrated eddy kinetic energy budget. Calculations of growth rate and wavelength have been described in the main text. Here we use an example to illustrate how the other metrics are defined and calculated.
The wave speed and phase are estimated by computing cross correlations in time and in the vertical coordinate, respectively. For wave speed, we take an alongshore profile of the perturbation cross-shore velocity $v^{\prime}$ at a $y$ location and find the spatial lag $\Delta x$ that has the maximum correlation between $v^{\prime}(x, t)$ and $v^{\prime}(x, t+\Delta t)\left(\Delta t=12 \mathrm{~h} ; v^{\prime}\right.$ is normalized to have an amplitude of 1$)$. The phase speed is $\Delta x / \Delta t$. We make estimates over a time period of 3 days centered at the maximal growth rate (e.g., same as $\sigma_{m}$ in Fig. 5 g) and for $y$ locations that span $1 / 4$ of the mean flow center. These estimates are then averaged to obtain a representative value.

A similar procedure is applied to determine the vertical wave phase. At the time of maximum growth rate, we computed the $x$ lag $(\Delta x)$ of maximum correlation between $v^{\prime}$ at the surface and $v^{\prime}$ at all other depths. We also estimate the wavelength as 2 times the distance between maximum and minimum $x$-lagged correlations in $v^{\prime}$. The wave phase in radians is then $2 \pi \Delta x / \lambda$. The vertical structure of wave amplitude is the root-meansquare of $v^{\prime}$ (i.e., in the alongshore direction) and is then averaged over a 3-day window and across the mean flow center (e.g., Fig. 10a).

Figure B1 provides an example for the above calculations. It also serves as a comparison to the Eady theory. For wave speed, Figs. B1a and B1b are the Hovmöller diagrams of the top and bottom $v^{\prime}$ at the mean flow center for a case with $\mathrm{Ri}=15$ (Fig. 5a1). The contour tilts clearly indicate wave propagation in the $+x$ direction. The contour slope at the surface (Fig. B1a) is steeper than a reference slope for mean flow advection (black line), indicating that the top wave propagates in the $-x$ direction, relative to the mean flow. Crosscorrelation estimates show that the apparent wave speed is $U / 2$, regardless the value of Ri (Fig. B1f; cases 2-5). The unstable waves in the model are therefore consistent with Eady's solution and Hoskins et al.'s (1985) interpretation. Counterpropagating top and bottom waves riding on the mean flow propagate at a phase-locking speed of $U / 2$.

For wave phase, a snapshot of $v^{\prime}$ along the $x-z$ plane shows a clear westward phase tilt (cf. Fig. B1c and Fig. 1d). Cross-correlation calculations in Fig. B1d yield a phase structure (black curve) that agrees well with Eady's most unstable mode (gray). This phase relation thus allows waves to amplify each other, manifested as growing instability.

In addition, the wave amplitude $v_{\mathrm{rms}}^{\prime}$ shows a boundary intensified structure consistent with the Eady solution (Fig. B1e). When Ri decreases, $v_{\mathrm{rms}}^{\prime}$ decays toward the interior at a shorter vertical scale. This behavior is consistent with the increasing nongeostrophic influences proposed by Nakamura (1988). 
(a) top v'

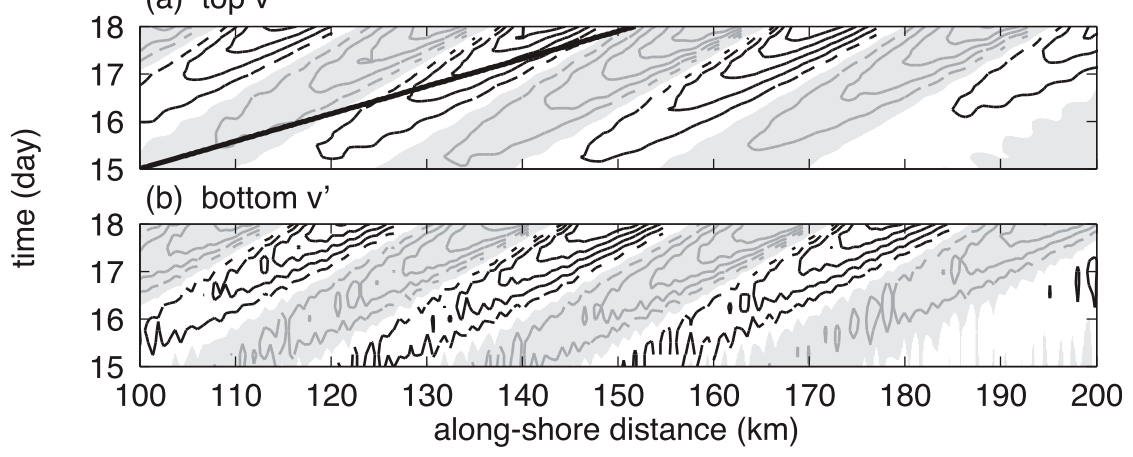

(c) $v^{\prime}(R i=15)$

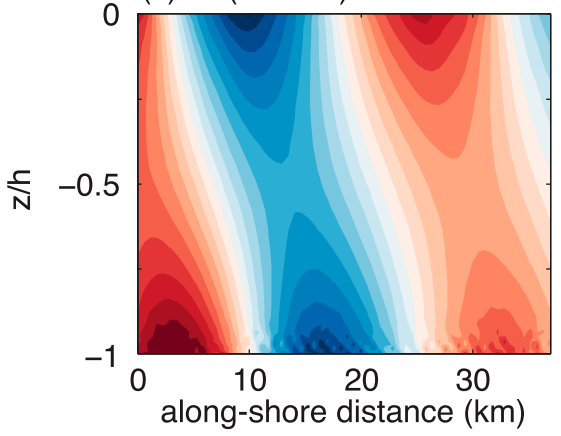

(d)
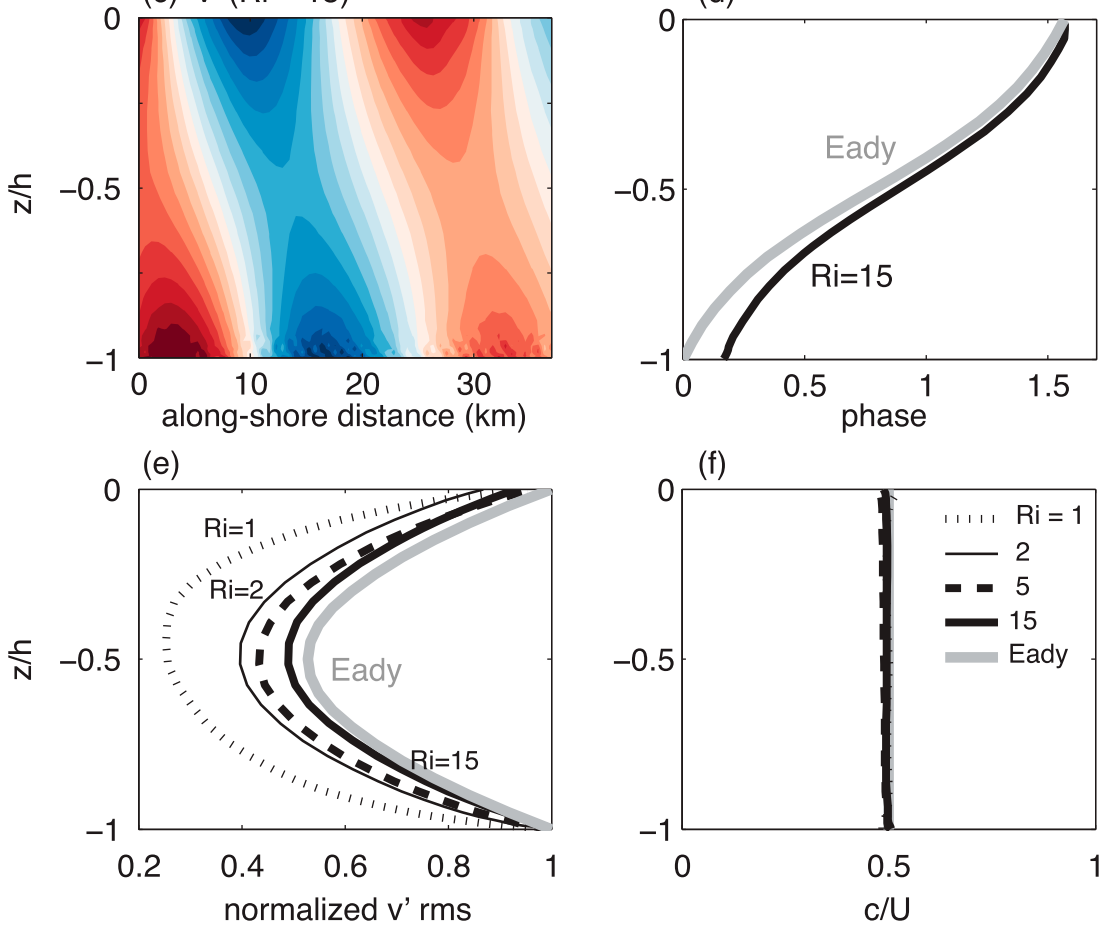

FIG. B1. Model estimates of unstable wave properties and comparisons with the Eady solutions. (a),(b) The Hovmöller diagram of the top and bottom wave signals $v^{\prime}$, taken in a 3-day window centered at the time of maximal growth rate (case 5 in Table $1 ; \mathrm{Ri}=15$; Fig. 5a1). The black reference line indicates the advective distance of the mean flow $U$. (c) A snapshot of $v^{\prime}$ in the $x-z$ plane, taken at maximal growth rate and along the mean flow center, for $\mathrm{Ri}=15$ that exhibits a westward phase tilt. Color shading represents the magnitude and direction of $v^{\prime}$. (d)-(f) The vertical structure of wave phase, amplitude, and apparent phase speed. The model results are labeled by different Ri values. The Eady solution is the thick gray curve. The model estimates of the wave properties are described in appendix B. Note in (e) that the case with a smaller Ri exhibits a shallower wave structure, consistent with Nakamura (1988). In (f), the unstable waves for all Ri values satisfy the phase-locking condition of $c=U / 2$.

Finally, to diagnose the energy source of the instability, we examine the volume integrated eddy kinetic energy $K_{E}$ budget. The derivation and notation follow von Storch et al. (2012). The balance equation for the eddy kinetic energy per volume $k_{e}\left[=\rho_{0}\left(\overline{u^{\prime 2}+v^{2}}\right) / 2\right]$ is

$$
\begin{aligned}
\frac{\partial k_{e}}{\partial t}+\nabla \cdot\left(k_{e} \overline{\mathbf{u}}\right)+\nabla \cdot\left[\frac{\rho_{0}}{2} \overline{\mathbf{u}^{\prime}\left(u^{\prime 2}+v^{\prime 2}\right)}\right]+\nabla \cdot \overline{p^{\prime} \mathbf{u}^{\prime}} \\
\left.=\left[-\rho_{0} \overline{u^{\prime} \mathbf{u}^{\prime}} \cdot \nabla \bar{u}-\rho_{0} \overline{v^{\prime} \mathbf{u}^{\prime}} \cdot \nabla \bar{v}\right]+\underset{\text { baroclinic }}{-g \overline{\rho^{\prime} w^{\prime}}}\right] \\
\quad \text { barotropic conversion } \\
+\left[\partial\left(\overline{\tau_{x}^{\prime} u^{\prime}}+\overline{\tau_{y}^{\prime} v^{\prime}}\right) / \partial z-\varepsilon\right], \\
\text { dissipation }
\end{aligned}
$$


where $\varepsilon=\overline{\tau_{x}^{\prime} \partial u^{\prime} / \partial z+\tau_{y}^{\prime} \partial v^{\prime} / \partial z}$. Integration over the entire volume of a reentry channel gives

$$
\frac{d K_{E}}{d t}=-C\left(K_{E}, K_{M}\right)+C\left(P_{E}, K_{E}\right)+D\left(K_{E}\right),
$$

where $K_{E}=\int k_{e} d V$, and the first and second terms on the right are the barotropic and baroclinic energy conversion

$$
\begin{aligned}
& C\left(K_{E}, K_{M}\right)=\int\left(\rho_{0} \overline{u^{\prime} \mathbf{u}^{\prime}} \cdot \nabla \bar{u}+\rho_{0} \overline{v^{\prime} \mathbf{u}^{\prime}} \cdot \nabla \bar{v}\right) d V, \\
& C\left(P_{E}, K_{E}\right)=\int-g \overline{\rho^{\prime} w^{\prime}} d V .
\end{aligned}
$$

They represent energy exchange between reservoirs of eddy and mean kinetic energy $\left[C\left(K_{E}, K_{M}\right)\right]$ and between eddy kinetic energy and eddy potential energy $\left[C\left(P_{E}, K_{E}\right)\right]$. Because we do not consider surface stresses in this study, we combine the work done by stresses and internal dissipation [last two terms in Eq. (B1)] into total energy dissipation

$$
D\left(K_{E}\right)=\int\left(\overline{\tau_{x}^{\prime} u^{\prime}}+\overline{\tau_{y}^{\prime} v^{\prime}}\right) d A-\int \varepsilon d V .
$$

\section{REFERENCES}

Allen, J. S., and P. A. Newberger, 1998: On symmetric instabilities in oceanic bottom boundary layers. J. Phys. Oceanogr., 28, 1131-1151, https://doi.org/10.1175/1520-0485(1998)028<1131: OSIIOB $>2.0 . \mathrm{CO} ; 2$.

Arnone, R. A., D. A. Wiesenburg, and K. D. Saunders, 1990: The origin and characteristics of the Algerian Current. J. Geophys. Res., 95, 1587-1598, https://doi.org/10.1029/JC095iC02p01587.

Badin, G., R. G. Williams, J. T. Holt, and L. J. Fernand, 2009: Are mesoscale eddies in shelf seas formed by baroclinic instability of tidal fronts? J. Geophys. Res., 114, C10021, https://doi.org/ 10.1029/2009JC005340

Barcilon, A., and W. Blumen, 1995: The Eady problem with linear horizontal shear. Dyn. Atmos. Oceans, 22, 115-133, https:// doi.org/10.1016/0377-0265(95)00404-X.

Barth, J. A., 1989: Stability of a coastal upwelling front: 2. Model results and comparison with observations. J. Geophys. Res., 94, 10 857-10 883, https://doi.org/10.1029/JC094iC08p10857.

Blokhina, M. D., and Y. D. Afanasyev, 2003: Baroclinic instability and transient features of mesoscale surface circulation in the Black Sea: Laboratory experiment. J. Geophys. Res., 108, 3322, https://doi.org/10.1029/2003JC001979.

Blumsack, S. L., and P. J. Gierasch, 1972: Mars: The effects of topography on baroclinic instability. J. Atmos. Sci., 29, 1081-1089, https:// doi.org/10.1175/1520-0469(1972)029<1081:MTEOTO>2.0.CO;2.

Bretherton, F. P., 1966: Baroclinic instability and short wavelength cut-off in terms of potential vorticity. Quart. J. Roy. Meteor. Soc., 92, 335-345, https://doi.org/10.1002/qj.49709239303.

Brink, K. H., 2012: Baroclinic instability of an idealized tidal mixing front. J. Mar. Res., 70, 661-688, https://doi.org/10.1357/ 002224012805262716.

, 2013: Instability of a tidal mixing front in the presence of realistic tides and mixing. J. Mar. Res., 71, 227-251, https:// doi.org/10.1357/002224013807719473.
_ 2016a: Continental shelf baroclinic instability. Part I: Relaxation from upwelling or downwelling. J. Phys. Oceanogr., 46, 551-568, https://doi.org/10.1175/JPO-D-15-0047.1.

— 2016b: Cross-shelf exchange. Annu. Rev. Mar. Sci., 8, 59-78, https://doi.org/10.1146/annurev-marine-010814-015717.

— , and S. J. Lentz, 2010: Buoyancy arrest and bottom Ekman transport. Part I: Steady flow. J. Phys. Oceanogr., 40, 621-635, https://doi.org/10.1175/2009JPO4266.1.

_ , and D. A. Cherian, 2013: Instability of an idealized tidal mixing front: Symmetric instabilities and frictional effects. J. Mar. Res., 71, 425-450, https://doi.org/10.1357/002224013812587582.

Chen, S. Y., and S. N. Chen, 2017: Generation of upwelling circulation under downwelling-favorable wind within bottomattached, buoyant coastal currents. J. Phys. Oceanogr., 47, 2499-2519, https://doi.org/10.1175/JPO-D-16-0271.1.

Durski, S. M., and J. S. Allen, 2005: Finite-amplitude evolution of instabilities associated with the coastal upwelling front. $J$. Phys. Oceanogr., 35, 1606-1628, https://doi.org/10.1175/JPO2762.1.

Eady, E. T., 1949: Long waves and cyclone waves. Tellus, 1, 33-52, https://doi.org/10.3402/tellusa.v1i3.8507.

Flagg, C. N., and R. C. Beardsley, 1978: On the stability of shelf water/slope water front south of New England. J. Geophys. Res., 83, 4623-4631, https://doi.org/10.1029/JC083iC09p04623.

Fox-Kemper, B., R. Ferrari, and R. Hallberg, 2008: Parameterization of mixed layer eddies. Part I: Theory and diagnosis. J. Phys. Oceanogr., 38, 1145-1165, https://doi.org/10.1175/ 2007JPO3792.1.

Fratantoni, P. S., and R. S. Pickart, 2003: Variability of the shelf break jet in the Middle Atlantic Bight: Internally or externally forced? J. Geophys. Res., 108, 3166, https://doi.org/10.1029/ 2002JC001326.

Gawarkiewicz, G., 1991: Linear-stability models of shelfbreak fronts. J. Phys. Oceanogr., 21, 471-488, https://doi.org/10.1175/ 1520-0485(1991)021<0471:LSMOSF $>2.0 . C O ; 2$.

Haidvogel, D. B., H. G. Arango, K. Hedstrom, A. Beckmann, P. Malanotte-Rizzoli, and A. F. Shchepetkin, 2000: Model evaluation experiments in the North Atlantic Basin: Simulations in nonlinear terrain-following coordinates. Dyn. Atmos. Oceans, 32, 239-281, https://doi.org/10.1016/S0377-0265(00)00049-X.

Haine, T. W. N., and J. Marshall, 1998: Gravitational, symmetric, and baroclinic instability of the ocean mixed layer. J. Phys. Oceanogr., 28, 634-658, https://doi.org/10.1175/1520-0485(1998) 028<0634:GSABIO > 2.0.CO;2.

Hetland, R. D., 2017: Suppression of baroclinic instabilities in buoyancy-driven flow over sloping bathymetry. J. Phys. Oceanogr., 47, 49-68, https://doi.org/10.1175/JPO-D-15-0240.1.

Holopainen, E. O., 1961: On the effect of friction in baroclinic waves. Tellus, 13, 363-367, https://doi.org/10.1111/j.21533490.1961.tb00097.x.

Hoskins, B. J., M. E. Mcintyre, and A. W. Robertson, 1985: On the use and significance of isentropic potential vorticity maps. Quart. J. Roy. Meteor. Soc., 111, 877-946, https://doi.org/ 10.1002/qj.49711147002.

Isachsen, P. E., 2011: Baroclinic instability and eddy tracer transport across sloping bottom topography: How well does a modified Eady model do in primitive equation simulations? Ocean Modell., 39, 183-199, https://doi.org/10.1016/j.ocemod.2010.09.007.

James, I. N., 1987: Suppression of baroclinic instability in horizontally sheared flows. J. Atmos. Sci., 44, 3710-3720, https:// doi.org/10.1175/1520-0469(1987)044<3710:SOBIIH >2.0.CO;2.

Johannessen, J. A., E. Svendsen, S. Sandven, O. M. Johannessen, and K. Lygre, 1989: Three-dimensional structure of mesoscale eddies in the Norwegian Coastal Current. J. Phys. Oceanogr., 
19, 3-19, https://doi.org/10.1175/1520-0485(1989)019<0003: TDSOME $>2.0 . \mathrm{CO} ; 2$.

Lozier, M. S., and M. S. C. Reed, 2005: The influence of topography on the stability of shelfbreak fronts. J. Phys. Oceanogr., 35, 1023-1036, https://doi.org/10.1175/JPO2717.1.

MacCready, P., and P. B. Rhines, 1991: Buoyant inhibition of Ekman transport on a slope and its effect on stratified spin-up. J. Fluid Mech., 223, 631-661, https://doi.org/10.1017/S0022112091001581.

McWilliams, J. C., E. Huckle, and A. F. Shchepetkin, 2009: Buoyancy effects in a stratified Ekman layer. J. Phys. Oceanogr., 39, 2581-2599, https://doi.org/10.1175/2009JPO4130.1.

Mechoso, C. R., 1981: Topographic influences on the general circulation of the Southern Hemisphere: A numerical experiment. Mon. Wea. Rev., 109, 2131-2139, https://doi.org/10.1175/ 1520-0493(1981)109<2131:TIOTGC>2.0.CO;2.

Nakamura, N., 1988: Scale selection of baroclinic instabilityEffects of stratification and nongeostrophy. J. Atmos. Sci., $\mathbf{4 5}$, 3253-3267, https://doi.org/10.1175/1520-0469(1988)045<3253: $\mathrm{SSOBIO}>2.0 . \mathrm{CO} ; 2$.

_ 1993: Momentum flux, flow symmetry, and the nonlinear barotropic governor. J. Atmos. Sci., 50, 2159-2179, https://doi.org/ 10.1175/1520-0469(1993)050<2159:MFFSAT>2.0.CO;2.

Pedlosky, J., 1979: Geophysical Fluid Dynamics. Springer, 624 pp.

_ 2 2016: Baroclinic instability over topography: Unstable at any wave number. J. Mar. Res., 74, 1-19, https://doi.org/10.1357/ 002224016818377595.

Pennel, R., A. Stegner, and K. Beranger, 2012: Shelf impact on buoyant coastal current instabilities. J. Phys. Oceanogr., 42, 39-61, https://doi.org/10.1175/JPO-D-11-016.1.

Pierrehumbert, R. T., and K. L. Swanson, 1995: Baroclinic instability. Annu. Rev. Fluid Mech., 27, 419-467, https:/doi.org/10.1146/ annurev.fl.27.010195.002223.

Pollard, R. T., P. B. Rhines, and R. Thompson, 1973: The deepening of the wind-mixed layer. Geophys. Fluid Dyn., 3, 381-404, https:// doi.org/10.1080/03091927208236105.

Qiu, B., N. Imasato, and T. Awaji, 1988: Baroclinic instability of buoyancy-driven coastal density currents. J. Geophys. Res., 93, 5037-5050, https://doi.org/10.1029/JC093iC05p05037.

Radko, T., D. Peixoto de Carvalho, and J. Flanaga, 2014: Nonlinear equilibration of baroclinic instability: The growth rate balance model. J. Phys. Oceanogr., 44, 1919-1940, https://doi.org/ 10.1175/JPO-D-13-0248.1.

Shchepetkin, A. F., and J. C. McWilliams, 2005: The Regional Oceanic Modeling System (ROMS): A split-explicit, free-surface, topography-following-coordinate oceanic model. Ocean Modell., 9, 347-404, https://doi.org/10.1016/j.ocemod. 2004.08.002.

Spall, M. A., 2004: Boundary currents and watermass transformation in marginal seas. J. Phys. Oceanogr., 34, 1197-1213, https://doi.org/10.1175/1520-0485(2004)034<1197: BCAWTI>2.0.CO;2.

_ 2007: Effect of sea surface temperature-wind stress coupling on baroclinic instability in the ocean. J. Phys. Oceanogr., 37, 1092-1097, https://doi.org/10.1175/JPO3045.1.
— , and L. N. Thomas, 2016: Downfront winds over buoyant coastal plumes. J. Phys. Oceanogr., 46, 3139-3154, https:// doi.org/10.1175/JPO-D-16-0042.1.

Stipa, T., 2004: On the sensitivity of coastal quasigeostrophic edge wave interaction to bottom boundary characteristics: Possible implications for eddy parameterizations. ArXiv, https://arxiv.org/ abs/physics/0401119.

Stone, P. H., 1966: On non-geostrophic baroclinic stability. J. Atmos. Sci., 23, 390-400, https://doi.org/10.1175/15200469(1966)023<0390:ONGBS > 2.0.CO;2.

__ 1971: Baroclinic stability under non-hydrostatic conditions. J. Fluid Mech., 45, 659-671, https://doi.org/10.1017/ S0022112071000260.

_, 1972: A simplified radiative-dynamical model for the static stability of rotating atmospheres. J. Atmos. Sci., 29, 405-418, https://doi.org/10.1175/1520-0469(1972)029<0405:ASRDMF> 2.0.CO;2.

Taylor, J. R., and S. Sarkar, 2008: Stratification effects in a bottom Ekman layer. J. Phys. Oceanogr., 38, 2535-2555, https:// doi.org/10.1175/2008JPO3942.1.

Thomas, L. N., A. Tandon, and A. Mahadevan, 2008: Submesoscale processes and dynamics. Ocean Modeling in an Eddying Regime, Geophys. Monogr., Vol. 177, Amer. Geophys. Union, 17-38, https://doi.org/10.1029/177GM04.

Vallis, G. K., 2017: Atmospheric and Oceanic Fluid Dynamics: Fundamentals and Large-Scale Circulation. 2nd ed. Cambridge University Press, 946 pp.

von Storch, J. S., C. Eden, I. Fast, H. Haak, D. Hernández-Deckers, E. Maier-Reimer, J. Marotzke, and D. Stammer, 2012: An estimate of the Lorenz energy cycle for the world ocean based on the STORM/NCEP simulation. J. Phys. Oceanogr., 42, 2185-2205, https://doi.org/10.1175/JPO-D-12-079.1.

Warner, J. C., C. R. Sherwood, H. G. Arango, and R. P. Signell, 2005: Performance of four turbulence closure models implemented using a generic length scale method. Ocean Modell., 8, 81-113, https://doi.org/10.1016/j.ocemod.2003.12.003.

, - - R. P. Signell, C. K. Harris, and H. G. Arango, 2008: Development of a three-dimensional, regional, coupled wave, current, and sediment-transport model. Comput. Geosci., 34, 1284-1306, https://doi.org/10.1016/j.cageo.2008.02.012.

Weingartner, T. J., S. Danielson, Y. Sasaki, V. Pavlov, and M. Kulakov, 1999: The Siberian Coastal Current: A wind- and buoyancy-forced Arctic coastal current. J. Geophys. Res., 104, 29 697-29713, https://doi.org/10.1029/1999JC900161.

Wenegrat, J. O., J. Callies, and L. N. Thomas, 2018: Submesoscale baroclinic instability in the bottom boundary layer. J. Phys. Oceanogr., 48, 2571-2592, https://doi.org/10.1175/JPO-D-17-0264.1.

Williams, G. P., and J. B. Robinson, 1974: Generalized Eady waves with Ekman pumping. J. Atmos. Sci., 31, 1768-1776, https://doi.org/10.1175/1520-0469(1974)031<1768:GEWWEP> 2.0.CO;2.

Zhang, W. F. G., and G. G. Gawarkiewicz, 2015: Length scale of the finite-amplitude meanders of shelfbreak fronts. J. Phys. Oceanogr., 45, 2598-2620, https://doi.org/10.1175/JPO-D-14-0249.1. 